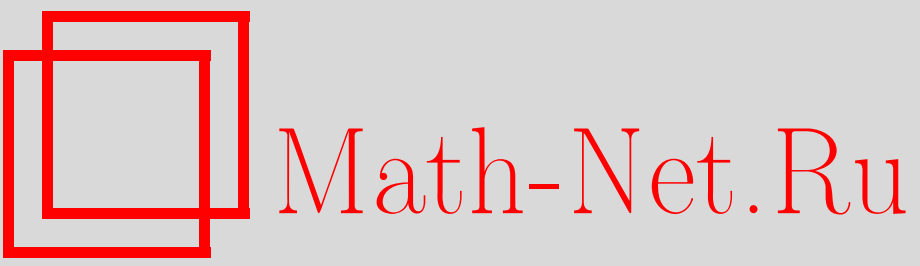

В. В. Белов, А. Ю. Трифонов, А. В. Шаповалов, Квазиклассическое траекторнокогерентное приближение для уравнения типа Хартри, ТМФ, 2002, том 130, номер 3, 460-492

DOI: https://doi.org/10.4213/tmf313

Использование Общероссийского математического портала Math-Net.Ru подразумевает, что вы прочитали и согласны с пользовательским соглашением

http://www.mathnet.ru/rus/agreement

Параметры загрузки :

IP: 107.22 .136 .117

26 апреля 2023 г., 10:00:51 
ТЕОРЕТИЧЕСКАЯ

И МАТЕМАТИЧЕСКАЯ

ФИЗИКА

Том 130, № 3

март, 2002

(C) 2002 г. $\quad$ В.В. Белов ${ }^{*}$, А. Ю. Трифонов ${ }^{\dagger}$, А.В. Шаповалов ${ }^{\ddagger}$

\section{КВАЗИКЛАССИЧЕСКОЕ ТРАЕКТОРНО-КОГЕРЕНТНОЕ ПРИБЛИЖЕНИЕ ДЛЯ УРАВНЕНИЯ ТИПА ХАРТРИ}

На основе комплексного метода ВКБ-Маслова построены квазиклассически сосредоточенные решения для уравнения типа Хартри. Формальные асимптотические по малому параметру $\hbar, \hbar \rightarrow 0$, решения задачи Коши для этого уравнения построены со степенной точностью $O\left(\hbar^{N / 2}\right)$, где $N \geqslant 3$ - любое натуральное число. Существенную роль при построении квазиклассически сосредоточенных решений играет выведенная в работе система уравнений Гамильтона-Эренфеста (система уравнений для средних и центрированных моментов). В классе квазиклассически сосредоточенных решений уравнения типа Хартри построена приближенная функция Грина и сформулирован нелинейный принцип суперпозиции.

\section{1. ВВЕДЕНИЕ}

В данной работе под уравнением типа Хартри понимается уравнение вида

$$
\left(-i \hbar \partial_{t}+\widehat{\mathcal{H}}_{\varkappa}\right) \Psi=0, \quad \Psi \in L_{2}\left(\mathbb{R}_{x}^{n}\right)
$$

где оператор типа Хартри $\widehat{\mathcal{H}}_{\varkappa}$ действует по формуле

$$
\widehat{\mathcal{H}}_{\varkappa} \Psi=(\widehat{\mathcal{H}}(t)+\varkappa \widehat{V}(t, \Psi)) \Psi
$$

Здесь

$$
\widehat{\mathcal{H}}(t)=\mathcal{H}(\hat{z}, t), \quad \widehat{V}(t, \Psi)=\int_{\mathbb{R}^{n}} d y \Psi^{*}(y, t) V(\hat{z}, \widehat{w}, t) \Psi(y, t),
$$

самосопряженные в $L_{2}$ операторы $\mathcal{H}(\hat{z}, t), V(\hat{z}, \widehat{w}, t)$ являются функциями от некоммутирующих операторов $\hat{z}=(-i \hbar \partial / \partial x, x), \widehat{w}=(-i \hbar \partial / \partial y, y), x, y \in \mathbb{R}^{n}$ и упорядочены

* Московский институт электроники и математики, Москва, Россия.

E-mail: belov@amath.msk.ru

† Томский политехнический университет, Томск, Россия. E-mail: trifonov@phtd.tpu.edu.ru

‡ Томский государственный университет, Томск, Россия. E-mail: shpv@phys.tsu.ru 
по Вейлю [1], [2], функция $\Psi^{*}$ - комплексно-сопряженная к функции $\Psi, \varkappa$ - вешественный параметр, $\hbar$ - "малый" параметр, $\hbar \in[0,1)$. Для операторов $\hat{z}$ и $\widehat{w}$ справедливы следующие коммутационные соотношения:

$$
\left[\hat{z}_{k}, \hat{z}_{j}\right]=\left[\widehat{w}_{k}, \widehat{w}_{j}\right]=i \hbar J_{k j}, \quad\left[\hat{z}_{k}, \widehat{w}_{j}\right]=0, \quad k, j=1, \ldots, 2 n,
$$

где $J=\left\|J_{k j}\right\|$ - стандартная симплектическая матрица размерности $2 n \times 2 n$,

$$
J=\left(\begin{array}{cc}
0 & -E_{n} \\
E_{n} & 0
\end{array}\right)
$$

Здесь $E_{n}$ - единичная $(n \times n)$-матрица, 0 - нулевая матрица порядка $n$.

В частном случае, когда вейлевские символы операторов в (1.3) имеют вид

$$
\mathcal{H}(z, t)=\frac{p^{2}}{2}+U(x, t), \quad p \in \mathbb{R}^{n}, \quad V(z, w, t)=V(x, y, t),
$$

уравнение (1.1) записывается как

$$
\hat{\mathcal{L}} \Psi=-i \hbar \frac{\partial \Psi}{\partial t}-\frac{\hbar^{2}}{2} \Delta \Psi+\left(U(x, t)+\varkappa \int_{\mathbb{R}^{n}} V(x, y, t)|\Psi(y, t)|^{2} d y\right) \Psi=0
$$

и называется в квантовой механике нестационарным уравнением типа Хартри во внешнем поле с потенциалом $U(x, t) \quad(V(x, y, t)$ - потенциал самосогласованного поля).

Это дифференшиальное уравнение с интегральной нелинейностью играет фундаментальную роль в квантовой теории и нелинейной оптике. В задачах квантовой механики и ядерной физики при исследовании взаимодействия систем многих частиц в приближении Хартри (см., например, [3]-[9]) потенциал $V(x, y, t)$ имеет, как правило, сингулярности, в частности, в обычном уравнении Хартри [10] - сингулярности кулоновского типа. Уравнение (1.4) с гладким интегральным ядром возникает при описании взаимодействия бозонов с формфактором в потенциале взаимодействия [11], фермионов в модели Тирринга [12], коллективных возбуждений в молекулярных цепочках [13], в сверхтекучих квантовых жидкостях и в неидеальном бозе-газе [14]. Это же уравнение появляется и в задачах квантовой теории нелинейных оптических явлений, например, при изучении "сжатого" [15] или “остановленного" [16] света, при описании распространения коротких и мошных импульсов в нелинейной среде с учетом вклада молекулярных колебаний в нелинейную поляризацию этой среды [17].

Математическая теория уравнения (1.4) (задача Коши) развита в работах [18]-[28]. Важные результаты получены также в спектральной теории этого уравнения [29]-[44]. Теория квазиклассического приближения при $\hbar \rightarrow 0$ для нелинейных уравнений типа уравнения (1.4) начала систематически разрабатываться в работах [45]-[47]. При этом предполагалось, что интегральное ядро в потенциале взаимодействия в (1.4) достаточно гладкое. Для этого случая в статье [46] построена формальная квазиклассическая 
асимптотика задачи Коши с быстроосциллируюшими начальными условиями. Для соответствуюших спектральных задач квазимоды, сосредоточенные вблизи точки, были впервые найдены в работах [46], [48], [49]. Асимптотические решения, локализованные в окрестности незамкнутых кривых, для стационарного уравнения (1.4) были построены в [50], [51]. Случай сингулярного ядра взаимодействия в квазиклассической асимптотике спектра этого уравнения активно исследовался в [47] и затем в [52]-[54]. Интересные результаты о квазимодах уравнения вида (1.4), сосредоточенных при $\hbar \rightarrow 0$ вблизи маломерных подмногообразий, были получены в [55], [56] на основе "сингулярного" варианта ВКБ-метода, разработанного в [54]. Наконец, отметим, что уравнения вида (1.1) (с симметричным символом интегрального "операторного" ядра в (1.3): $V(z, w, t)=V(w, z, t))$ играют важную роль при построении асимптотических решений для $N$-частичного уравнения Шредингера при $N \rightarrow \infty$ [57], [58].

В данной работе для уравнения (1.1) (с гладкими вейлевскими символами в оператоpe (1.2)) мы строим локализованные асимптотические при $\hbar \rightarrow 0$ решения - "квазисолитоны", обладающие рядом свойств, присущих уединенным волнам.

В линейном случае - для уравнения типа Шредингера (уравнение (1.1) при $\varkappa=0$ ) - подобные локализованные асимптотические решения были построены в [59]- [62] и получили название "квазиклассически сосредоточенных" решений (или состояний). В работах [61]-[65] такие состояния были построены для уравнений Клейна-Гордона и Дирака в произвольном электромагнитном поле и для уравнений Шредингера и Дирака во внешнем неабелевом поле с калибровочной группой $S U(2)$. Квазиклассически сосредоточенные состояния являются обобшением хорошо известных (сжатых) когерентных состояний [66]-[72] на случай (линейных) уравнений квантовой механики в произвольных внешних полях. На их основе в работах [61], [62], [73]-[75] был развит новый ковариантный подход в квазиклассическом приближении для нерелятивистских уравнений квантовой механики. Суть этого подхода состоит в том, что в классе квазиклассически сосредоточенных состояний средние значения наблюдаемых приближенно (с любой степенью точности $\left.O\left(\hbar^{(N+1) / 2}\right), \hbar \rightarrow 0, N \geqslant 0\right)$ определяются по решению конечномерной аппроксимации порядка $N$ системы обыкновенных дифференциальных уравнений относительно квантовых средних базисного набора наблюдаемых теории (в случае, например, уравнения типа Шредингера этот базисный набор является универсальной обертывающей алгебры Гейзенберга-Вейля). Такая система для уравнения Шредингера была впервые получена в статье [73] (для более обших случаев - в работах [64], [65], [74]-[77]) и названа системой Гамильтона-Эренфеста. В работах [76], [77] было доказано, что она является пуассоновой системой относительно (вырожденной) нелинейной скобки Дирака.

Оказывается, что при построении локализованных асимптотических решений для уравнения типа Хартри (по аналогии с линейным случаем мы их будем называть квазиклассически сосредоточенными решениями или состояниями, имея в виду квантово-механическую версию уравнения (1.1)) на основе ковариантного подхода удается линеаризовать это уравнение с любой степенной точностью по параметру $\hbar, \hbar \rightarrow 0$, и при- 
менить затем квазиклассические методы, развитые для линейных уравнений, в частности, комплексный метод ВКБ (метод комплексного ростка Маслова) [48], [78].

Для уединенных волн (“квазисолитонов") характерно проявление свойств, присущих частицам. Для “квазисолитонов” - квазиклассически сосредоточенных состояний уравнения типа Хартри - эти свойства представлены ниже динамической системой обыкновенных дифференциальных уравнений относительно "квантовых" средних $X(t, \hbar), P(t, \hbar)$ операторов координат $\hat{x}$ и импульсов $\hat{p}$ и центрированных моментов высших порядков. Такая система ранее в литературе не рассматривалась. В пределе $\hbar \rightarrow 0$ "центр тяжести" такого "квазисолитона" движется в фазовом пространстве по траектории этой динамической системы: в каждый момент времени квазиклассически сосредоточенное состояние эффективно сосредоточено в окрестности точки $X(t, 0)($ в $x$-представлении) и в окрестности точки $P(t, 0)$ (в $p$-представлении). Эту систему ${ }^{1)}$ относительно квантовых средних для уравнения типа Хартри мы, так же как и в линейном случае, называем системой Гамильтона-Эренфеста.

Отметим, наконец, следующий важный факт. В отличие от (линейного) уравнения типа Шредингера в самой конструкции квазиклассически сосредоточенных состояний уравнения типа Хартри существенно используются решения системы ГамильтонаЭренфеста. Проиллюстрируем этот факт на примере эволюции многомерного (сжатого) когерентного состояния. В этом случае в уравнении (1.4) внешний потенциал $U(x, t)$ и потенциал самосогласованного поля $V(x, y, t)$ - гладкие (бесконечно дифференцируемые) фрункци по всем своим аргументам, растущие на бесконечности (при $|x| \rightarrow \infty$, $|y| \rightarrow \infty)$ не быстрее некоторой степени относительно $|x|,|y|$ равномерно по $t \in \mathbb{R}$. Здесь и далее |.| обозначает евклидову норму векторов соответствующей размерности.

Для уравнения (1.4) рассмотрим задачу Коши с начальным условием в виде гауссова пакета,

$$
\left.\Psi\right|_{t=0}=\Psi_{0}(x, \hbar)=N_{0} e^{\frac{i}{\hbar}\left\langle p_{0}, x-x_{0}\right\rangle} e^{\frac{i}{\hbar}\left\langle\left(x-x_{0}\right), B_{0}\left(x-x_{0}\right)\right\rangle},
$$

где $\left(p_{0}, x_{0}\right)$ - произвольная точка фазового пространства $\mathbb{R}_{p, x}^{2 n}, B_{0}$ - комплексная симметричная матрица размерности $n \times n$ с положительной мнимой частью,

$$
B_{0}^{t}=B_{0}, \quad \operatorname{Im} B_{0}=\frac{B_{0}-B_{0}^{+}}{2 i}>0,
$$

нормировочная постоянная $N_{0}=(\pi \hbar)^{-n / 4}\left(\operatorname{det} \operatorname{Im} B_{0}\right)^{1 / 4}$. Здесь и далее индексы $t$ и + означают транспонирование и эрмитово сопряжение, соответственно, а скобки $\langle$,$\rangle - ев-$ клидово скалярное произведение векторов.

Уравнению (1.4) сопоставим систему обыкновенных дифференциальных уравнений (систему Гамильтона-Эренфеста порядка $N=2$ ) относительно фазовых переменных $(p, x) \in \mathbb{R}_{p, x}^{2 n}$ и вешественной $(2 n \times 2 n)$-матрищы дисперсий $\Delta_{2}$, составленной из блоков - $(n \times n)$-матриц $\sigma_{p p}=\sigma_{p p}^{t}, \sigma_{x x}=\sigma_{x x}^{t}, \sigma_{p x}=\sigma_{x p}^{t}$,

$$
\Delta_{2}=\left(\begin{array}{cc}
\sigma_{p p} & \sigma_{p x} \\
\sigma_{x p} & \sigma_{x x}
\end{array}\right)
$$

\footnotetext{
1) Свойство гамильтоновости этой системы является предметом отдельного исследования.
} 
Эта система имеет вид

$$
\left\{\begin{array}{l}
\dot{x}=p \\
\dot{p}=-\left.\nabla_{x} \varphi^{\varkappa}(x, y, t)\right|_{y=x}-\left.\frac{1}{2} \nabla_{x} \operatorname{Sp}\left(\sigma_{x x}\left(\varphi_{x x}^{\varkappa}(x, y, t)+\varphi_{y y}^{\varkappa}(x, y, t)\right)\right)\right|_{y=x} \\
\dot{\Delta}_{2}=J M \Delta_{2}-\Delta_{2} M J
\end{array}\right.
$$

где

$$
\varphi^{\varkappa}(x, y, t)=U(x, t)+\varkappa V(x, y, t)
$$

и $(2 n \times 2 n)$-матрица

$$
M=M^{\varkappa}(x, t)=\left(\begin{array}{cc}
E_{n} & 0 \\
0 & \left.\varphi_{x x}^{\varkappa}(x, y, t)\right|_{y=x}
\end{array}\right) .
$$

Здесь $\varphi_{x x}^{\varkappa}, \varphi_{y y}^{\varkappa}-(n \times n)$-матрицы вторых производных,

$$
\varphi_{y y}^{\varkappa}(x, y, t)=\left(\frac{\partial^{2} \varphi^{\varkappa}}{\partial y_{i} \partial y_{j}}\right)=\varkappa\left(\frac{\partial^{2} V}{\partial y_{i} \partial y_{j}}\right)(x, y, t) .
$$

Начальное условие $\Psi_{0}(x, \hbar)(1.5)$ индуцирует начальные данные для системы $(1.7)$, (1.8)

$$
\left.x\right|_{t=0}=x_{0},\left.\quad p\right|_{t=0}=p_{0}
$$

и

$$
\left.\Delta\right|_{t=0}=\Delta_{2}^{\Psi_{0}}(\hbar)
$$

где $\Delta_{2}^{\Psi_{0}}(\hbar)$ - матрица квантовых центрированных моментов второго порядка для операторов $(-i \hbar \partial / \partial x, x)=\hat{z}$ в состоянии $\Psi_{0}(x, \hbar)$. Таким образом, имеем

$$
\sigma_{x x}(0)=\sigma_{x x}\left(\Psi_{0}\right), \quad \sigma_{p p}(0)=\sigma_{p p}\left(\Psi_{0}\right), \quad \sigma_{p x}(0)=\sigma_{p x}\left(\Psi_{0}\right), \quad \sigma_{x p}(0)=\sigma_{p x}^{t}(0)
$$

где

$$
\sigma_{A B}\left(\Psi_{0}\right)=\frac{1}{2}(\langle(\hat{A} \widehat{B}+\widehat{B} \hat{A})\rangle-\langle\hat{A}\rangle\langle\widehat{B}\rangle)
$$

и через $\langle\hat{A}\rangle$ обозначено среднее значение оператора $\hat{A}$ с символом $A$ в состоянии $\Psi_{0}$ : $\langle\hat{A}\rangle=\left\langle\Psi_{0}|\hat{A}| \Psi_{0}\right\rangle$, где $\langle\mid\rangle$ - скалярное произведение в $L_{2}\left(\mathbb{R}^{n}\right)$. Нетрудно вычислить квантовые средние в состоянии $\Psi_{0}(x, \hbar)(1.5)$,

$\sigma_{x x}(0)=\frac{\hbar}{2} D_{0}^{-1}, \quad \sigma_{p p}(0)=\frac{\hbar}{4}\left(B_{0} D_{0}^{-1} B_{0}^{*}+B_{0}^{*} D_{0}^{-1} B_{0}\right), \quad \sigma_{p x}(0)=\frac{\hbar}{4}\left(B_{0}+B_{0}^{*}\right) D_{0}^{-1}$, где $D_{0}=\operatorname{Im} B_{0}$ и знак * означает комплексное сопряжение.

Обозначим через $y(t, \hbar)=\left(P(t, \hbar), X(t, \hbar), \Delta_{2}(t, \hbar)\right)$ решение задачи Коши $(1.7),(1.8)$ и $(1.7 \mathrm{a}),(1.8 \mathrm{a})$ на интервале $[0, T], T>0$, гладко зависящее от малого параметра $\hbar$, 
$\hbar \rightarrow 0$, входящего в начальное условие $(1.8 \mathrm{a}): \Delta_{2}^{\Psi_{0}}(\hbar)=O(\hbar)$. Оказывается, что для построения главного члена квазиклассической асимптотики $(\hbar \rightarrow 0)$ исходной квантовой задачи $(1.4),(1.5)$ достаточно найти главный член разложения в ряд регулярной теории возмущений по $\hbar \rightarrow 0$ решения соответствуюшей задачи Коши для системы Гамильтона-Эренфеста (1.7), (1.8),

$$
y(t, \hbar)=y^{0}(t, \hbar)+\hbar y^{1}(t, \hbar)+\cdots
$$

где $y^{0}(t, \hbar)=\left(P^{0}(t), X^{0}(t), \Delta^{0}\left(t, \hbar, \Psi_{0}\right)\right)$, причем $\Delta^{0}(t, \hbar)=O(\hbar), \hbar \rightarrow 0$ и $P^{0}(0)=p_{0}$, $X^{0}(0)=x_{0}, \Delta^{0}(0, \hbar)=\Delta_{2}^{\Psi_{0}}(\hbar)$. Очевидно, что с точностью до членов порядка $O(\hbar)$, $\hbar \rightarrow 0$, система $(1.7),(1.8)$ расцепляется в том смысле, что матрица “дисперсий" $\Delta^{0}(t, \hbar)$ не влияет на фазовую траекторию $\left(P_{\varkappa}^{0}(t), X_{\varkappa}^{0}(t)\right), t \in[0, T]$, которая определяется решением системы уравнений

$$
\left\{\begin{array}{l}
\dot{x}=p \\
\dot{p}=-\left.\nabla_{x} \varphi^{\varkappa}(x, y, t)\right|_{y=x}
\end{array}\right.
$$

с начальными данными $(1.7 \mathrm{a})$, a $\Delta^{0}(t, \hbar)$ - решение задачи Коши

$$
\begin{aligned}
& \frac{d}{d t} \Delta^{0}=J M^{\varkappa}(t) \Delta^{0}-\Delta^{0} M^{\varkappa}(t) J, \\
& \left.\Delta^{0}\right|_{t=0}=\Delta_{2}^{\Psi_{0}}(\hbar),
\end{aligned}
$$

где с учетом (1.9)

$$
M^{\varkappa}(t)=M^{\varkappa}\left(X_{\varkappa}^{0}(t), t\right)=\left(\begin{array}{cc}
E_{n} & 0 \\
0 & \varphi_{x x}^{\varkappa}\left(X_{\varkappa}^{0}(t), X_{\varkappa}^{0}(t), t\right)
\end{array}\right) .
$$

Решение последней задачи Коши для нелинейного уравнения (1.11) заменой

$$
\Delta^{0}\left(t, \hbar, \Psi_{0}\right)=\Phi(t) \Delta_{2}^{\Psi_{0}}(\hbar) \Phi(t)
$$

сводится к построению матрицы Коши $\Phi(t), \Phi(0)=E_{2 n}$, для линейной гамильтоновой системы

$$
\dot{a}=J M^{\varkappa}(t) a, \quad a \in \mathbb{C}^{2 n}, \quad a=(w, z), \quad w, z \in \mathbb{C}^{n} .
$$

Очевидно, что при $\varkappa=0$ система (1.10) является стандартной гамильтоновой системой с функцией Гамильтона $\mathcal{H}(p, x, t)=p^{2} / 2+U(x, t)$, а система $(1.14)$ - системой в вариациях [48], линеаризацией этой системы на ньютоновской классической траектории $\left(P^{0}(t)=\dot{X}^{0}(t), X^{0}(t)\right)$. При $\varkappa \neq 0$ будем называть (допуская некоторую некорректность в терминологии) систему (1.10) и (1.14) системой Гамильтона и системой в вариациях с самодействием, соответственно.

Так же как и в линейной теории $(\varkappa=0)$ (см., например, [48], [62]), существенную роль в конструкции локализованных асимптотических решений задачи (1.4), (1.5) играют 
комплексные решения системы в вариациях с самодействием. Обозначим через $B(t)$ и $C(t)$ комплексные $(n \times n)$-матрицы - "импульсные" и “координатные" составляюшие матричного решения системы (1.14),

$$
\left(\begin{array}{c}
\dot{B} \\
\dot{C}
\end{array}\right)=J M^{\varkappa}(t)\left(\begin{array}{c}
B \\
C
\end{array}\right) \Longleftrightarrow \begin{aligned}
& \dot{C}=B, \\
& \dot{B}=-\varphi_{x x}^{\varkappa}\left(X_{\varkappa}^{0}(t), X_{\varkappa}^{0}(t), t\right) C,
\end{aligned}
$$

удовлетворяющие начальным данным

$$
\left.B\right|_{t=0}=B_{0},\left.\quad C\right|_{t=0}=E_{n}
$$

В силу свойства гамильтоновости системы уравнений (1.15) и свойств матрицы $B_{0}(1.6)$ матрица $C(t), t \in[0, T]$, невырожденна, так что определена симметричная матрица $B C^{-1}(t)$ с положительной мнимой частью, $\operatorname{Im} B C^{-1}(t)>0$ (детали доказательства см., например, в [48]).

Определим функцию

$$
\Psi(x, t, \hbar)=N_{0} e^{\frac{i}{\hbar} S^{\varkappa}(x, t, \hbar)}(\operatorname{det} C(t))^{-1 / 2},
$$

где комплексная фаза $S^{\varkappa}(x, t)$ имеет вид

$$
\begin{aligned}
S^{\varkappa}(x, t, \hbar)= & \int_{0}^{t}\left\{\left\langle P_{\varkappa}^{0}(\tau), \dot{X}_{\varkappa}^{0}(\tau)\right\rangle-\mathcal{H}\left(P_{\varkappa}^{0}(\tau), X_{\varkappa}^{0}(\tau), \tau\right)+\varkappa V_{y}\left(X_{\varkappa}^{0}(\tau), X_{\varkappa}^{0}(\tau), \tau\right)+\right. \\
& \left.+\varkappa \frac{1}{2} \operatorname{Sp}\left(\sigma_{x x}\left(\tau, \hbar, \psi_{0}\right) V_{y y}^{\prime \prime}\left(X_{\varkappa}^{0}(\tau), X_{\varkappa}^{0}(\tau), \tau\right)\right)\right\} d \tau+ \\
& +\left\langle P_{\varkappa}^{0}(t), x-X_{\varkappa}^{0}(t)\right\rangle+\left\langle x-X_{\varkappa}^{0}(t), B C^{-1}(t)\left(x-X_{\varkappa}^{0}(t)\right)\right\rangle .
\end{aligned}
$$

Здесь $\mathcal{H}(p, x, t)=p^{2} / 2+U(x, t), \sigma_{x x}\left(t, \hbar, \psi_{0}\right)$ - нижний правый блок матрицы "дисперсий" $\Delta^{0}\left(t, \hbar, \psi_{0}\right)$.

ТЕОРЕМА 1.1. Пусть выполнень сформулированные выше условия на потенциальи $U(x, t), V(x, y, t)$ и пусть на интервале $[0, T]$ существует гладкое решение задачи (1.10), (1.7a). Тогда при $t \in[0, T]$ функиия $\Psi(x, t, \hbar)$ (1.17) является формальным асимптотическим $\left(\bmod \hbar^{3 / 2}, \hbar \rightarrow 0\right)$ решением задачи $(1.4),(1.5)$,

$$
\hat{\mathcal{L}}(\Psi(x, t, \hbar))=O\left(\hbar^{3 / 2}\right),\left.\quad \Psi(x, t, \hbar)\right|_{t=0}=\Psi_{0}(x, \hbar),
$$

где через $O\left(\hbar^{3 / 2}\right)$ обозначена гладкая функиия $g(x, t, \hbar)$ - “невязка” уравнения (1.2) в $L_{2}$-норме, $\max _{0 \leqslant t \leqslant T}\|g(x, t, \hbar)\|_{L_{2}}=O\left(\hbar^{3 / 2}\right)$ nри $\hbar \rightarrow 0$.

Доказательство теоремы следует из общих конструкций (см. ниже раздел 5). 
ЗАмЕчАнИЕ 1.1. В рассмотренном выше примере $(n \times n)$-блок $\sigma_{x x}\left(t, \hbar, \Psi_{0}\right)$ матрищы “дисперсий” $\Delta^{0}\left(t, \hbar, \Psi_{0}\right)$, определяюший в фазе $S^{\varkappa}(x, t, \hbar)(1.18)$ поправку порядка $\hbar$, $\hbar \rightarrow 0$, несложно вычислить через матрицу $C(t)$, если матрицу Коши системы (1.14) записать в виде

$$
\Phi(t)=\left(\begin{array}{ll}
B(t) & B^{*}(t) \\
C(t) & C^{*}(t)
\end{array}\right)\left(\begin{array}{ll}
B_{0} & B_{0}^{*} \\
E_{n} & E_{n}
\end{array}\right)^{-1},
$$

воспользоваться формулой (1.13) и явным видом матрицы дисперсий $\Delta_{2}^{\Psi_{0}}(\hbar)$. В результате несложных, но громоздких вычислений получим

$$
\sigma_{x x}\left(t, \hbar, \Psi_{0}\right)=\frac{\hbar}{4}\left(C(t) D_{0}^{-1} C^{+}(t)+C^{*}(t) D_{0}^{-1} C^{t}(t)\right)
$$

где $D_{0}=\operatorname{Im} B_{0}$

Таким образом, квазиклассически сосредоточенное состояние $\Psi(x, t, \hbar)(1.17)$, приближенно $\left(\bmod \hbar^{3 / 2}\right.$ при $\left.\hbar \rightarrow 0\right)$ описываюшее эволюцию начального сжатого когерентного состояния для уравнения вида (1.4), определяется в силу теоремы 1.1 решениями двух систем обыкновенных дифференциальных уравнений: нелинейной системы Гамильтона с самодействием (1.10) и соответствуюшей ей линейной гамильтоновой системы в вариациях (1.14).

Специфика уравнения типа Хартри, в котором нелинейность присутствует только под знаком интеграла, проявляется в том, что оно обладает рядом свойств, присуших линейным уравнениям. Так, в частности, удалось показать, что в классе квазиклассически сосредоточенных решений для этого уравнения (с заданной степенью точности по $\hbar$, $\hbar \rightarrow 0)$ справедлив нелинейный принцип суперпозиции.

В рамках рассматриваемого подхода построены асимптотические решения задачи Коши для этого уравнения, а также найден оператор эволюции в классе траекторно-сосредоточенных функций со степенной точностью до любого конечного порядка по малому параметру $\sqrt{\hbar}$ при $\hbar \rightarrow 0$.

Подчеркнем, что всюду в данной работе речь идет о построении формальных асимптотических решений уравнения типа Хартри с невязкой, норма которой имеет сколь угодно малую оценку по параметру $\hbar, ~ \hbar \rightarrow 0$. Обоснование этих асимптотик на конечных временах $t \in[0, T], T=$ const, представляет собой отдельную непростую математическую задачу ${ }^{2}$. Эта задача связана с получением априорных оценок для решения нелинейного уравнения типа Хартри, равномерных по параметру $\hbar \in(0,1]$. В данной работе такая задача не рассматривается. Из эвристических соображений, приведенных в работе [47], вытекает, что оценка разности между точным и формально построенным асимптотическим решениями, по-видимому, может быть получена с использованием методов, развитых в работах [47], [81].

План дальнейшего изложения следующий. Во втором разделе дана постановка задачи о построении квазиклассических асимптотик в классе траекторно-сосредоточенных

\footnotetext{
2) Формальная асимптотика по $t, t \rightarrow \infty$, и фиксированном $\hbar=1$ для уравнения (1.4) была построена в случае специальных классов потенциалов в ряде работ (см., например, [79], [80]).
} 
функций и рассмотрены их простейшие свойства. В разделе 3 получена система Гамильтона-Эренфеста, описываюшая "частицеподобные" свойства квазиклассически сосредоточенных решений уравнения типа Хартри. В разделе 4 уравнение типа Хартри линеаризовано с помошью решений системы Гамильтона-Эренфеста и получена система ассоциированных линейных уравнений, определяюших асимптотическое решение исходной задачи. В пятом разделе с точностью $O\left(\hbar^{3 / 2}\right)$ исследуется динамика фоковских состояний уравнения типа Хартри и получен главньй член квазиклассической асимптотики этого уравнения. Квазиклассически сосредоточенные решения (обший случай) для уравнения Хартри построены с точностью до $O\left(\hbar^{3 / 2}\right)$ в разделе 6 , а с любой степенной точностью по параметру $\sqrt{\hbar}$ при $\hbar \rightarrow 0-$ в разделе 7 . В восьмом разделе найден приближенный оператор эволюции уравнения типа Хартри. Там же дано обоснование нелинейного принципа суперпозиции в классе квазиклассически сосредоточенных решений. В приложении дано обоснование основных свойств траекторно-сосредоточенных функций.

\section{2. ПОСТАНОВКА ЗАДАЧИ В КЛАССЕ КВАЗИКЛАССИЧЕСКИ СОСРЕДОТОЧЕННЫХ ФУНКЦИЙ}

Построение асимптотических решений уравнения типа Хартри проведем при следуюшем предположении о свойствах вейлевских символов операторов $\mathcal{H}(\hat{z}, t)$ и $V(\hat{z}, \widehat{w}, t)$ в (1.1):

ПРЕДПОЛОЖЕНИЕ 2.1. Функции $\mathcal{H}(z, t)$ и $V(z, w, t)$ являются $C^{\infty}$-гладкими функциями и вместе со всеми своими производными по $z$ и $w$ растут при $|z| \rightarrow \infty$ и $|w| \rightarrow \infty$ не быстрее, чем полином, равномерно по $t \in \mathbb{R}$, т.е. для любых мультииндексов $\alpha, \beta, \mu, \nu \in$ $\mathbb{Z}_{+}^{2 n}$ и $T>0$ сушествуют постоянные $C_{\beta}^{\alpha}(T)$ и $C_{\beta \nu}^{\alpha \mu}(T)$ такие, что выполняются неравенства

$$
\begin{aligned}
\left|z^{\alpha} \frac{\partial^{|\beta|} \mathcal{H}(z, t)}{\partial z^{\beta}}\right| & \leqslant C_{\beta}^{\alpha}(T), \\
\left|z^{\alpha} w^{\mu} \frac{\partial^{|\beta+\nu|} V(z, w, t)}{\partial z^{\beta} \partial w^{\nu}}\right| & \leqslant C_{\beta \nu}^{\alpha \mu}(T), \quad z, w \in \mathbb{R}^{2 n}, \quad 0 \leqslant t \leqslant T .
\end{aligned}
$$

Здесь

$$
\begin{gathered}
\frac{\partial^{|\alpha|} V(z)}{\partial z^{\alpha}}=\frac{\partial^{|\alpha|} V(z)}{\partial z_{1}^{\alpha_{1}} \partial z_{2}^{\alpha_{2}} \ldots \partial z_{2 n}^{\alpha_{2 n}}}, \quad \alpha_{j} \in \mathbb{Z}_{+}, \quad j=1, \ldots, 2 n \\
\alpha=\left(\alpha_{1}, \alpha_{2}, \ldots, \alpha_{2 n}\right), \quad|\alpha|=\alpha_{1}+\alpha_{2}+\cdots+\alpha_{2 n}, \quad z^{\alpha}=z_{1}^{\alpha_{1}} z_{2}^{\alpha_{2}} \ldots z_{2 n}^{\alpha_{2 n}} .
\end{gathered}
$$

Перейдем к описанию класса функций, в котором мы будем искать локализованные асимптотические решения уравнения (1.1). Функции этого класса, сингулярно зависяшие от малого параметра $\hbar$, представляют собой обобщение понятия уединенной волны. 
Они зависят от произвольной фазовой траектории $z=Z(t, \hbar) \in \mathbb{R}_{p, x}^{2 n}, t \in \mathbb{R}^{1}$, и вешественнозначной функции $S(t, \hbar)$ (аналога классического действия в линейном случае при $\varkappa=0)$. В пределе $\hbar \rightarrow 0$ функции этого класса сосредоточены в окрестности точки, движушейся вдоль заданной фазовой кривой $z=Z(t, 0)$. Такие функции хорошо известны в квантовой механике. К ним, в частности, относятся когерентные и “сжатые" состояния квантовых систем с квадратичным гамильтонианом [70], [71].

Обозначим этот класс функций через $\mathcal{P} t(Z(t, \hbar), S(t, \hbar))$ и определим его следуюшим образом:

$$
\begin{aligned}
\mathcal{P}_{\hbar}^{t} & =\mathcal{P}_{\hbar}^{t}(Z(t, \hbar), S(t, \hbar))= \\
& =\left\{\Phi: \Phi(x, t, \hbar)=\varphi\left(\frac{\Delta x}{\sqrt{\hbar}}, t, \sqrt{\hbar}\right) \exp \left[\frac{i}{\hbar}(S(t, \hbar)+\langle P(t, \hbar), \Delta x\rangle)\right]\right\},
\end{aligned}
$$

где комплекснозначная функция $\varphi(\xi, t, \sqrt{\hbar})$ принадлежит пространству Шварца $\mathbb{S}$ по переменной $\xi \in \mathbb{R}^{n}$, гладким образом зависит от $t$ и регулярно зависит от $\sqrt{\hbar}$ при $\hbar \rightarrow 0$. В соотношении (2.1) $\Delta x=x-X(t, \hbar)$, а вешественная функция $S(t, \hbar)$ и $2 n$-мерная вектор-функция $Z(t, \hbar)=(P(t, \hbar), X(t, \hbar))$, характеризующие класс $\mathcal{P}_{\hbar}^{t}(Z(t, \hbar), S(t, \hbar))$, peгулярно зависят от $\sqrt{\hbar}$ в окрестности $\hbar=0$ и подлежат определению при $t>0$, причем $S(0, \hbar)=0, Z(0, \hbar)=z_{0}=\left(p_{0}, x_{0}\right)$ - произвольная точка фазового пространства $\mathbb{R}_{p x}^{2 n}$.

Функции, принадлежащие классу $\mathcal{P}_{\hbar}^{t}$, в любой фиксированный момент времени $t \in$ $\mathbb{R}^{1}$ сосредоточеньи в пределе $\hbar \rightarrow 0$ в окрестности точки, лежашей на фазовой кривой $z=Z(t, 0), t \in \mathbb{R}^{1}$ (точньй смысл этого свойства устанавливается ниже формулами $(2.6),(2.7))$. Поэтому функции класса $\mathcal{P}_{\hbar}^{t}$ естественно назвать траекторно-сосредоточенныци функииями при $\hbar \rightarrow 0$. В определение класса траекторно-сосредоточенных функций входят в качестве свободных "параметров" фазовая траектория $Z(t, \hbar)$ и скалярная функция $S(t, \hbar)$. Оказывается, что эти "параметры" однозначно определяют$\mathrm{cr}^{3)}$ из системы Гамильтона-Эренфеста (см. разд. 4), отвечающей нелинейному $(\varkappa \neq 0)$ гамильтониану уравнения (1.1). Отметим, что в предельном случае $\varkappa=0$ для линейного уравнения типа Шредингера вектор-функция $Z(t, 0)$ - главньй член разложения по $\hbar \rightarrow 0$ - определяет фазовую траекторию гамильтоновой системы с классическим гамильтонианом $\mathcal{H}(p, x, t)$, а функция $S(t, 0)$ является классическим действием вдоль этой траектории. В частности, в этом случае классу $\mathcal{P}_{\hbar}^{t}$ принадлежат хорошо известные динамические (сжатые) когерентные состояния квантовых систем с квадратичными гамильтонианами при выборе амплитуды $\varphi$ в (2.1) в форме гауссовой экспоненты,

$$
\varphi(\xi, t)=\exp \left[\frac{i}{2}\langle\xi, Q(t) \xi\rangle\right] f(t),
$$

где $Q(t)$ - комплексная симметричная матрица с положительной мнимой частью, а временной множитель $f(t)$ определяется формулой

$$
f(t)=\sqrt[4]{\operatorname{det} \operatorname{Im} Q(t)} \exp \left[-\frac{i}{2} \int_{0}^{t} \operatorname{Sp} \operatorname{Re} Q(\tau) d \tau\right]
$$

\footnotetext{
3) Результаты, полученные в этом разделе, так же как и в разделе 3 , не зависят от явного вида функций $Z(t, \hbar)$ и $S(t, \hbar)$.
} 
(см. [61], [62]). Здесь и далее через $\mathrm{Sp} A$ обозначен след матрицы $A$.

Рассмотрим основные свойства функций из класса $\mathcal{P}_{\hbar}^{t}(Z(t, \hbar), S(t, \hbar))$, обоснование которых дано в приложении.

1. Для функиий класса $\mathcal{P}_{\hbar}^{t}(Z(t, \hbar), S(t, \hbar))$ справедливь следующие асимптотические оченки для чентрированных моментов $\Delta_{\alpha}(t, \hbar)$ порядка $|\alpha|, \quad \alpha \in \mathbb{Z}_{+}^{2 n}$ :

$$
\begin{gathered}
\Delta_{\alpha}(t, \hbar)=\Delta_{\alpha}^{\Phi}(t, \hbar)=\frac{\left\langle\Phi\left|\{\Delta \hat{z}\}^{\alpha}\right| \Phi\right\rangle}{\|\Phi\|^{2}}=O\left(\hbar^{|\alpha| / 2}\right), \quad \hbar \rightarrow 0, \quad|\alpha| \neq 0, \\
\Delta_{\alpha}(t, \hbar)=1, \quad|\alpha|=0 .
\end{gathered}
$$

Через $\{\Delta \hat{z}\}^{\alpha}$ обозначен оператор с вейлевским символом $(\Delta z)^{\alpha}$,

$$
\Delta z=z-Z(t, \hbar)=(\Delta p, \Delta x), \quad \Delta p=p-P(t, \hbar), \quad \Delta x=x-X(t, \hbar) .
$$

Обозначим символом $\widehat{O}\left(\hbar^{k}\right), k \geqslant 0$, оператор $\widehat{F}$ такой, что для любой функции $\Phi$, принадлежащей $\mathcal{P}_{\hbar}^{t}(Z(t, \hbar), S(t, \hbar))$, справедлива асимптотическая оценка

$$
\frac{\|\widehat{F} \Phi\|}{\|\Phi\|}=O\left(\hbar^{k}\right), \quad \hbar \rightarrow 0 .
$$

2. Для функиий, принадлежащих $\mathcal{P}_{\hbar}^{t}(Z(t, \hbar), S(t, \hbar))$, справедливь следующие асимптотические оченки:

$$
\begin{gathered}
\left\{-i \hbar \partial_{t}-\dot{S}(t, \hbar)+\langle P(t, \hbar), \dot{X}(t, \hbar)\rangle+\langle\dot{Z}(t, \hbar), J \Delta \hat{z}\rangle\right\}=\widehat{O}(\hbar), \\
\{\Delta \hat{z}\}^{\alpha}=\widehat{O}\left(\hbar^{|\alpha| / 2}\right), \quad \alpha \in \mathbb{Z}_{+}^{2 n}, \quad \hbar \rightarrow 0
\end{gathered}
$$

и, в частности,

$$
\Delta \hat{x}_{k}=\widehat{O}(\sqrt{\hbar}), \quad \Delta \hat{p}_{j}=\widehat{O}(\sqrt{\hbar}), \quad k, j=1, \ldots, n .
$$

Следуюшее свойство придает точный смысл понятию сосредоточенности при $\hbar \rightarrow 0$ в окрестности точки на фазовой траектории для функций из класса $\mathcal{P}_{h}^{t}$.

3. Для любой функции $\Phi(x, t, \hbar) \in \mathcal{P}_{\hbar}^{t}(Z(t, \hbar), S(t, \hbar))$ справедливы предельнье соотношения

$$
\begin{aligned}
& \lim _{\hbar \rightarrow 0} \frac{1}{\|\Phi\|^{2}}|\Phi(x, t, \hbar)|^{2}=\delta(x-X(t, 0)), \\
& \lim _{\hbar \rightarrow 0} \frac{1}{\|\tilde{\Phi}\|^{2}}|\tilde{\Phi}(p, t, \hbar)|^{2}=\delta(p-P(t, 0)),
\end{aligned}
$$

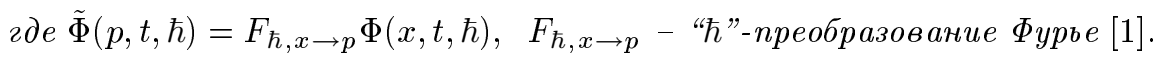


4. Обозначим через $\langle\hat{L}(t)\rangle$ среднее значение самосопряженного в $L_{2}\left(\mathbb{R}_{x}^{n}\right)$ оператора $\hat{L}(t), \quad t \in \mathbb{R}^{1}$, вычисленное по функции $\Phi(x, t, \hbar) \in \mathcal{P}_{\hbar}^{t}$. Тогда для любой функиии $\Phi(x, t, \hbar) \in \mathcal{P}_{\hbar}^{t}(Z(t, \hbar), S(t, \hbar))$ и любого оператора $\hat{A}(t, \hbar)$, вейлевский символ которого $A(z, t, \hbar)$ удовлетворяет предположсению 2.1 , справедливо равенство

$$
\lim _{\hbar \rightarrow 0}\langle\hat{A}(t, \hbar)\rangle=\lim _{\hbar \rightarrow 0} \frac{1}{\|\Phi\|^{2}}\langle\Phi(x, t, \hbar)|\hat{A}(t, \hbar)| \Phi(x, t, \hbar)\rangle=A(Z(t, 0), t, 0) .
$$

По аналогии с линейной теорией [62] дадим следующее определение.

ОПРедЕЛЕниЕ 2.1. Решение $\Phi(x, t, \hbar)$ уравнения $(1.1)$ в классе функций $\mathcal{P}_{\hbar}^{t}$ назовем квазиклассически сосредоточенным при $\hbar \rightarrow 0$ на фазовой траектории $Z(t, \hbar)$.

Предельный характер условий (2.6), (2.7) и асимптотический характер оценок (2.2)(2.5), справедливых в классе траекторно-сосредоточенных функций, позволяют строить квазиклассически сосредоточенные решения уравнения типа Хартри не точно, а приближенно. При этом $L_{2}$-норма погрешности имеет порядок $\hbar^{\alpha}, \alpha>1$, при $\hbar \rightarrow 0$ на любом конечном временном интервале $[0, T]$. Обозначим такое приближенное решение через $\Psi_{\mathrm{as}}=\Psi_{\mathrm{as}}(x, t, \hbar)$. Оно удовлетворяет следуюшей задаче:

$$
\begin{aligned}
& {\left[-i \hbar \frac{\partial}{\partial t}+\widehat{\mathcal{H}}(t)+\varkappa \widehat{V}\left(t, \Psi_{\mathrm{as}}\right)\right] \Psi_{\mathrm{as}}=O\left(\hbar^{\alpha}\right),} \\
& \Psi_{\mathrm{as}} \in \mathcal{P}_{\hbar}^{t}(Z(t, \hbar), S(t, \hbar), \hbar), \quad t \in[0, T],
\end{aligned}
$$

где через $O\left(\hbar^{\alpha}\right)$ обозначена функция $g^{(\alpha)}(x, t, \hbar)$ - “невязка" уравнения (1.1) в норме $L_{2}$,

$$
\max _{0 \leqslant t \leqslant T}\left\|g^{(\alpha)}(x, t, \hbar)\right\|=O\left(\hbar^{\alpha}\right), \quad \hbar \rightarrow 0 .
$$

Функцию $\Psi_{\text {аs }}(x, t, \hbar)$, удовлетворяюшую задаче (2.9)-(2.11), будем также называть $к в a-$ зиклассически сосредоточенным решением $\left(\bmod \hbar^{\alpha}, \hbar \rightarrow 0\right)$ уравнения типа Хартри (1.1).

Основной целью работы является построение квазиклассически сосредоточенных решений уравнения типа Хартри с любой степенью точности по степеням малого параметра $\sqrt{\hbar}, \hbar \rightarrow 0$, т.е. функций $\Psi_{\text {as }}(x, t, \hbar)=\Psi^{(N)}(x, t, \hbar)$, удовлетворяющих задаче (2.9)-(2.11) по $\bmod \left(\hbar^{(N+1) / 2}\right)$, где $N \geqslant 2$ - любое натуральное число.

Таким образом, квазиклассически сосредоточенные решения $\Psi^{(N)}(x, t, \hbar)$ уравнения типа Хартри приближенно описывают эволюцию начального состояния $\Psi_{0}(x, \hbar)$, если оно выбрано в классе траекторно-сосредоточенных функций $\mathcal{P}_{\hbar}^{0}$ :

$$
\left.\Psi(x, t, \hbar)\right|_{t=0}=\Psi_{0}(x, \hbar), \quad \Psi_{0} \in \mathcal{P}_{\hbar}^{0}\left(z_{0}, 0\right) .
$$

Функции из класса $\mathcal{P}_{\hbar}^{0}$ имеют вид

$$
\Psi_{0}(x, \hbar)=\exp \left\{\frac{i}{\hbar}\left[\left\langle p_{0}, x-x_{0}\right\rangle\right]\right\} \varphi_{0}\left(\frac{x-x_{0}}{\sqrt{\hbar}}, \sqrt{\hbar}\right), \quad \varphi_{0}(\xi, \sqrt{\hbar}) \in \mathbb{S}\left(\mathbb{R}_{\xi}^{n}\right),
$$


где $z_{0}=\left(p_{0}, x_{0}\right)$ - произвольная точка фазового пространства $\mathbb{R}_{p x}^{2 n}$.

Важными частными случаями начальных условий вида (2.13) являются, например, следующие:

а) гауссов пакет, если $\varphi_{0}(\xi, \sqrt{\hbar})=e^{-\langle\xi, A \xi\rangle / 2}$, где вешественная $(n \times n)$-матрица $A$ положительно определена и симметрична;

б) фоковские состояния, если $\varphi_{0}(\xi, \sqrt{\hbar})=\varphi_{\nu}(\xi)=e^{i\langle\xi, Q \xi\rangle / 2} H_{\nu}(\sqrt{\operatorname{Im} Q} \xi)$, где комплексная $(n \times n)$-матрица $Q$ симметрична и имеет положительно определенную мнимую часть $\operatorname{Im} Q$, а $H_{\nu}(\eta), \eta \in \mathbb{R}^{n},-$ многомерные полиномы Эрмита мультииндекса $\nu=\left(\nu_{1}, \ldots, \nu_{n}\right)$ [82]. В частности, при $\nu=0$ функция $\Psi_{0}(x, \hbar)$ определяет сжатое когерентное состояние (1.5).

\section{3. СИСТЕМА УРАВНЕНИЙ ГАМИЛЬТОНА-ЭРЕНФЕСТА}

Символы $\mathcal{H}(z, t)$ и $V(z, w, t)$ удовлетворяют условиям предположения 2.1. Поэтому оператор $\mathcal{H}(\hat{z}, t)$ в $(1.3)$ самосопряжен относительно скалярного произведения $\langle\Psi \mid \Phi\rangle$ в пространстве $L_{2}\left(\mathbb{R}_{x}^{n}\right)$, а оператор $V(\hat{z}, \widehat{w}, t)$ - относительно скалярного произведения $L_{2}\left(\mathbb{R}_{x y}^{2 n}\right)$,

$$
\langle\Psi(t) \mid \Phi(t)\rangle_{\mathbb{R}^{2 n}}=\int_{\mathbb{R}^{2 n}} d x d y \Psi^{*}(x, y, t, \hbar) \Phi(x, y, t, \hbar) .
$$

Следовательно, квадрат нормы точных решений уравнения (1.1) сохраняется, т.е. $\|\Psi(t)\|^{2}=\|\Psi(0)\|^{2}$, и для средних значений оператора $\hat{A}(t)=A(\hat{z}, t)$, вычисленных на этих решениях, справедливо равенство

$$
\frac{d}{d t}\langle\hat{A}(t)\rangle=\left\langle\frac{\partial \hat{A}(t)}{\partial t}\right\rangle+\frac{i}{\hbar}\langle[\hat{\mathcal{H}}, \hat{A}(t)]\rangle+\frac{i \varkappa}{\hbar}\left\langle\int d y \Psi^{*}(y, t, \hbar)[\hat{A}(t), V(\hat{z}, \widehat{w}, t)] \Psi(y, t, \hbar)\right\rangle,
$$

где $[\hat{A}, \widehat{B}]=\hat{A} \widehat{B}-\widehat{B} \hat{A}-$ коммутатор операторов $\hat{A}$ и $\widehat{B}$. По аналогии с линейным $(\varkappa=0)$ уравнением Шредингера в квантовой механике равенство (3.1) назовем уравнением Эренфеста для средних значений оператора $\hat{A}(t)$, отвечаюшим уравнению типа Хартри.

Мы предполагаем, что для уравнения (1.1) существуют точные (или отличаюшиеся от них на величину $O\left(\hbar^{\infty}\right)$ ) решения в классе траекторно-сосредоточенных функций. Выпишем уравнения Эренфеста (3.1) для средних значений операторов $\hat{z},\{\Delta \hat{z}\}^{\alpha}$, вычисленных по таким (траекторно-сосредоточенным) решениям уравнения (1.1). Будем при этом использовать правила композиции вейлевских символов [1]

$$
C(z)=A\left(\stackrel{2}{z}+\frac{i \hbar}{2} J \frac{1}{\partial z}\right) B(z)=B\left(\stackrel{2}{z}-\frac{i \hbar}{2} J \frac{1}{\partial z}\right) A(z),
$$

где $C(z)$ - символ оператора $\widehat{C}=\hat{A} \widehat{B}$ и знаки 1 и 2 над оператором указывают очередность его действия (напомним, что $\hat{z}=(\hat{p}, \hat{x}), Z(t, \hbar)=(P(t, \hbar), X(t, \hbar)), \Delta \hat{z}=\hat{z}-$ $Z(t, \hbar))$. После громоздких, но несложных расчетов, аналогичных линейному случаю 
$(\varkappa=0)$ (детали этих вычислений можно найти в [61], [62], [75]), получим, ограничиваясь моментами порядка $N$, следующую систему обыкновенных дифференциальных уравнений:

$$
\begin{aligned}
\dot{z}= & \sum_{|\mu|=0}^{N} \frac{1}{\mu !} J\left(\mathcal{H}_{z \mu}(z, t) \Delta_{\mu}+\tilde{\varkappa} \sum_{|\nu|=0}^{N} \frac{1}{\nu !} V_{z \mu \nu}(z, t) \Delta_{\nu} \Delta_{\mu}\right) \\
\dot{\Delta}_{\alpha}= & \sum_{|\mu+\gamma|=0}^{N}(-i \hbar)^{|\gamma|-1} \frac{\left[(-1)^{\left|\gamma_{p}\right|}-(-1)^{\left|\gamma_{x}\right|}\right] \alpha ! \beta ! \theta(\alpha-\gamma) \theta(\beta-\gamma)}{\gamma !(\alpha-\gamma) !(\beta-\gamma) ! \mu !} \times \\
& \times\left(\mathcal{H}_{\mu}(z, t)+\tilde{\varkappa} \sum_{|\nu|=0}^{N} \frac{1}{\nu !} V_{\mu \nu}(z, t) \Delta_{\nu}\right) \Delta_{\alpha-\gamma+J \beta-J \gamma}- \\
& -\sum_{k=1}^{2 n} \dot{z}_{k} \alpha_{k} \Delta_{\alpha(k)}, \quad|\alpha|=1,2, \ldots, N .
\end{aligned}
$$

Здесь $\Delta_{\alpha}=\Delta_{\alpha_{1} \alpha_{2} \ldots \alpha_{2 n}}$ вектор длины $(2 n)^{|\alpha|}$, компоненты которого $\Delta_{\alpha}^{a}$ занумерованы $|\alpha|$-мерным мультииндексом $a=\left(a_{1}, \ldots, a_{i}, \ldots, a_{|\alpha|}\right), \quad 1 \leqslant a_{i} \leqslant 2 n$, и обозначено: $\tilde{\varkappa}=\varkappa\left\|\Psi_{0}(x, \hbar)\right\|^{2}, \Psi_{0}(x, \hbar)$ - начальная функция из $(2.12), \alpha, \mu, \nu, \beta, \gamma$ - мультииндексы длины $2 n$,

$$
\begin{gathered}
\mathcal{H}_{\mu}(z, t)=\frac{\partial^{|\mu|} \mathcal{H}(z, t)}{\partial z^{\mu}}, \quad V_{\mu \nu}(z, t)=\left.\frac{\partial^{|\mu+\nu|} V(z, w, t)}{\partial z^{\mu} \partial w^{\nu}}\right|_{w=z}, \\
\mathcal{H}_{z \mu}(z, t)=\partial_{z} \mathcal{H}_{\mu}(z, t), \\
\alpha=\left(\alpha_{p}, \alpha_{x}\right), \quad J \alpha=\left(\alpha_{x}, \alpha_{p}\right), \quad \theta(\alpha-\beta)=\prod_{k=1}^{2 n} \theta\left(\alpha_{k}-\beta_{k}\right), \\
\alpha(k)=\left(\alpha_{1}-\delta_{1, k}, \ldots, \alpha_{2 n}-\delta_{2 n, k}\right),
\end{gathered}
$$

где $\theta(x)$ - $\theta$-рункция и $J \gamma=\left(\gamma_{x}, \gamma_{p}\right), \gamma_{x}=\left(\gamma_{n+1}, \ldots, \gamma_{2 n}\right), \gamma_{p}=\left(\gamma_{1}, \ldots, \gamma_{n}\right)$ - компоненты мультииндекса $\gamma=\left(\gamma_{1}, \ldots, \gamma_{n}, \gamma_{n+1}, \ldots, \gamma_{2 n}\right)$.

Так же как и в линейной теории $(\varkappa=0)$, систему (3.2) назовем системой Гамильтона-Эренфеста порядка $N, N=0,1,2, \ldots$ В силу оценок (2.2) эта система с точки зрения вычисления квантовых средних эквивалентна решению нелинейного уравнения (1.1) в классе траекторно-сосредоточенных функций, если начальные данные для (3.2) задать как приближенные по $\bmod O\left(\hbar^{(N+1) / 2}\right), \hbar \rightarrow 0$, значения квантово-механических средних от операторов $\hat{z}, \widehat{\Delta z^{\alpha}}$ в начальном состоянии $\Psi_{0}(x, \hbar)(2.12)$, а именHO

$$
\left.z\right|_{t=0}=z^{N}(\hbar),\left.\quad \Delta_{\alpha}\right|_{t=0}=\Delta_{\alpha}^{N}(\hbar), \quad 1 \leqslant|\alpha| \leqslant N
$$

где

$$
\begin{aligned}
& \Delta_{\alpha}^{\Psi_{0}}(\hbar)=\frac{\left\langle\Psi_{0}\left|\widehat{\Delta z}^{\alpha}\right| \Psi_{0}\right\rangle}{\left\|\Psi_{0}\right\|^{2}}=\Delta_{\alpha}^{N}(\hbar)+O\left(\hbar^{(N+1) / 2}\right) \\
& z^{\Psi_{0}}(\hbar)=\frac{\left\langle\Psi_{0}|\hat{z}| \Psi_{0}\right\rangle}{\left\|\Psi_{0}\right\|^{2}}=z^{N}(\hbar)+O\left(\hbar^{(N+1) / 2}\right), \quad N=0,1,2, \ldots
\end{aligned}
$$


Отметим, что в силу системы Гамильтона-Эренфеста порядка $N$ при $N \geqslant 0$ для переменных $\Delta_{\alpha}$, где $|\alpha|=1$, имеем $\dot{\Delta}_{\alpha}=0$ и, следовательно, для задачи Коши $(3.2),(3.3)$ $\Delta_{\alpha}(t, \hbar)=\mathrm{const}=\Delta_{\alpha}^{N}(\hbar)$ при любом $N$. Если теперь начальная траекторно-сосредоточенная функция $\Psi_{0}(x, \hbar)$ удовлетворяет условию траекторной когерентности (см. об этом подробнее в работах [61], [62])

$$
z^{\Psi_{0}}(\hbar)=z_{0} \quad \text { для любого } \hbar \in(0,1]
$$

где $z_{0}$ - произвольная точка фазового пространства, $z_{0}=\left(p_{0}, x_{0}\right) \in \mathbb{R}_{p, x}^{2 n}$, то тогда, очевидно, $\Delta_{\alpha}(t, \hbar)=\Delta_{\alpha}^{\Psi_{0}}(\hbar, N)=0$ при $|\alpha|=1$. Таким образом, системы ГамильтонаЭренфеста порядков $N=0$ и $N=1$ совпадают и имеют вид

$$
\dot{z}=J\left[\mathcal{H}_{z}(z, t)+\left.\tilde{\varkappa} V_{z}(z, \omega, t)\right|_{\omega=z}\right]
$$

Выпишем в этом же случае (при вьполнении условия (3.5)) систему Гамильтона-Эренфеста порядка $N=2$, представив набор переменных $\Delta_{\alpha},|\alpha|=2$, в виде $(2 n \times 2 n)$-матрицы “дисперсий" $\Delta_{2}=\left(\Delta_{2}^{a_{1} a_{2}}\right), 1 \leqslant a_{1}, a_{2} \leqslant 2 n$. Тогда из $(3.2)$ имеем

$$
\begin{aligned}
\dot{z} & =J \partial_{z}\left(1+\frac{1}{2}\left\langle\partial_{z}, \Delta_{2} \partial_{z}\right\rangle+\frac{1}{2}\left\langle\partial_{\omega}, \Delta_{2} \partial_{\omega}\right\rangle\right)\left(\mathcal{H}(z, t)+\left.\tilde{\varkappa} V(z, \omega, t)\right|_{\omega=z}\right), \\
\dot{\Delta}_{2} & =J M \Delta_{2}-\Delta_{2} M J,
\end{aligned}
$$

где $(2 n \times 2 n)$-матрица

$$
M=\mathcal{H}_{z z}(z, t)+\left.\tilde{\varkappa} V_{z z}(z, \omega, t)\right|_{\omega=z}
$$

ЗАмечаниЕ 3.1. Условию (3.5) удовлетворяет, например, сжатое когерентное состояние $\Psi_{0}(x, \hbar)$ вида (1.5). Поэтому в теореме 1.1 при конструкции асимптотик была использована система Гамильтона-Эренфеста порядка $N=2$ в форме (3.7).

\section{4. ЛИНЕАРИЗАЦИЯ УРАВНЕНИЯ ТИПА ХАРТРИ}

Центральным моментом рассматриваемого подхода является линеаризация (с любой степенной точностью по параметру $\hbar \rightarrow 0$ ) уравнения типа Хартри в классе траекторно-сосредоточенных функций. Существенную роль при этом играет решение задачи Коши (3.2), (3.3) для системы Гамильтона-Эренфеста порядка $N$. Решение этой задачи обозначим через $y_{\Psi_{0}}^{N}(t, \hbar)=\left(Z^{N}(t, \hbar), \Delta_{\alpha}^{N}(t, \hbar),|\alpha| \leqslant N\right)$.

Обозначим (см. (2.2))

$$
\begin{aligned}
z^{\Psi}(t, \hbar) & =\frac{1}{\|\Psi(t, \hbar)\|^{2}}\langle\Psi(t, \hbar)|\widehat{w}| \Psi(t, \hbar)\rangle \\
\Delta_{\alpha}^{\Psi}(t, \hbar) & =\frac{1}{\|\Psi(t, \hbar)\|^{2}}\left\langle\Psi(t, \hbar)\left|\{\Delta \widehat{w}\}^{\alpha}\right| \Psi(t, \hbar)\right\rangle
\end{aligned}
$$


и разложим "ядро" оператора $\widehat{V}(t, \Psi)$ в ряд Тейлора по степеням операторов $\Delta \widehat{w}=$ $\widehat{w}-z^{\Psi}(t, \hbar)$,

$$
V(\hat{z}, \widehat{w}, t)=\left.\sum_{|\alpha|=0}^{N} \frac{1}{\alpha !} \frac{\partial^{|\alpha|} V(\hat{z}, w, t)}{\partial w^{\alpha}}\right|_{w=z^{\Psi}(t, \hbar)}\{\Delta \widehat{w}\}^{\alpha}+\widehat{R}_{N+1},
$$

где остаточный член, оператор $\widehat{R}_{N+1}=R_{N+1}\left(\hat{z}, z^{\Psi}(t, \hbar), \Delta \widehat{w}^{N+1}\right)$ на функциях $\Psi \in \mathcal{P}_{n}^{t}$ допускает ${ }^{4)}$ следуюшую оценку по $\hbar \rightarrow 0:$

$$
\left\|\Psi(x) \int_{\mathbb{R}^{n}} \Psi^{*}(y) \widehat{R}_{N+1} \Psi(y) d y\right\|_{L_{2}\left(\mathbb{R}_{x}^{n}\right)}=O\left(\hbar^{(N+1) / 2}\right) .
$$

Подставив это разложение в уравнение (1.1), на функциях $\Psi \in \mathcal{P}_{\hbar}^{t}$ с учетом оценки (4.3) получим

$$
\begin{aligned}
\hat{L}^{(N)}(t, \Psi) \Psi & =\left[-i \hbar \partial_{t}+\mathcal{H}(\hat{z}, t)+\left.\widetilde{\varkappa} \sum_{|\alpha|=0}^{N} \frac{1}{\alpha !} \frac{\partial^{|\alpha|} V(\hat{z}, w, t)}{\partial w^{\alpha}}\right|_{w=z^{\Psi}(t, \hbar)} \Delta_{\alpha}^{\Psi}(t, \hbar)\right] \Psi= \\
& =O\left(\hbar^{(N+1) / 2}\right) .
\end{aligned}
$$

Воспользуемся теперь результатами предыдушего раздела: "квантовые" моменты до порядка $N$ включительно на решении $\Psi(x, t, \hbar) \in \mathcal{P}_{\hbar}^{t}$ приближенно, с точностью до моментов $\Delta_{\alpha}^{\Psi}(t, \hbar),|\alpha|>N$, определяются решением системы Гамильтона-Эренфеста порядка $N$, и, следовательно, с учетом асимптотических оценок $(2.2)$ для коэффициентов $z^{\Psi}(t, \hbar)$ и $\Delta_{\alpha}^{\Psi}(t, \hbar)$ в $(4.4)$ справедливы (с любой степенью точности по $\left.\hbar \rightarrow 0\right)$ равенства

$$
\begin{aligned}
z^{\Psi}(t, \hbar) & =Z^{N}(t, \hbar)+O\left(\hbar^{(N+1) / 2}\right), \\
\Delta_{\alpha}^{\Psi}(t, \hbar) & =\Delta_{\alpha}^{N}(t, \hbar)+O\left(\hbar^{(N+1) / 2}\right), \quad|\alpha| \leqslant N .
\end{aligned}
$$

Подставив эти разложения коэффициентов в оператор $\hat{L}^{(N)}(t, \Psi)$, получим, что

$$
\hat{L}^{(N)}(t, \Psi)=\hat{L}^{(N)}\left(t, \Psi_{0}\right)+\widehat{O}_{N+1}(\hbar),
$$

где оператор $\widehat{O}_{N+1}(\hbar)=\widehat{O}\left(\hbar^{(N+1) / 2}\right)$ в смысле оценки $(2.3)$, а $\hat{L}^{(N)}\left(t, \Psi_{0}\right)$ - линейныц й оператор типа оператора ШІредингера имеет вид

$$
\hat{L}^{(N)}\left(t, \Psi_{0}\right)=-i \hbar \partial_{t}+\mathcal{H}(\hat{z}, t)+\left.\tilde{\varkappa} \sum_{|\alpha|=0}^{N} \frac{1}{\alpha !} \frac{\partial^{|\alpha|} V(\hat{z}, w, t)}{\partial w^{\alpha}}\right|_{w=Z^{N}(t, \hbar)} \Delta_{\alpha}^{N}(t, \hbar)
$$

с коэффициентами, зависящими от $Z^{N}(t, \hbar)$ и $\Delta_{\alpha}^{N}(t, \hbar),|\alpha| \leqslant N$, и определяемыми в силу системы (3.2) заданной начальной функцией $\Psi_{0}(x, \hbar)(2.12)$.

\footnotetext{
4) Это нетрудно показать, основываясь на теории оценок для “ $\hbar$ ”-псевдодифференциальных операторов с вейлевскими символами, удовлетворяющими предположению 2.1 (см., например, [1], [81]).
} 
Таким образом, замена (4.5) квантовых средних значений операторов $\widehat{w}$ и $\widehat{\Delta w}\}^{\alpha}$ на решения системы Гамильтона-Эренфеста порядка $N$ в уравнении (4.4) линеаризует уравнение $(1.1)$ с точностью до $O\left(\hbar^{(N+1) / 2}\right)$, где $N \geqslant 2$. Следовательно, для построения квазиклассически сосредоточенных состояний $\bmod \hbar^{(N+1) / 2}$ нелинейного уравнения (1.1) достаточно построить с этой же точностью асимптотику решения линейного уравнения

$$
\hat{L}^{(N)}\left(t, \Psi_{0}\right) \Phi=0, \quad \Phi \in \mathcal{P}_{\hbar}^{t} .
$$

ОПРЕДЕЛЕНИЕ 4.1. Уравнение вида (4.8) при заданном $\Psi_{0}(2.12)$ будем называть уравнением Хартри в траекторно-когерентном приближении или линейным ассоциированным уравнением Шредингера порядка $N$ для уравнения (1.1), а его (произвольное) решение в классе функций $\mathcal{P}_{\hbar}^{t}$ будем обозначать ${ }^{5)}$ через $\Phi=\Phi\left(x, t, \hbar, \Psi_{0}\right)$.

Очевидным является следующее важное утверждение.

УТВЕРЖДЕНИЕ 4.1. Если функиия $\Phi^{(N)}\left(x, t, \hbar, \Psi_{0}\right) \in \mathcal{P}_{\hbar}^{t}$, асимптотическое $\left(\right.$ с точностью $\left.O\left(\hbar^{(N+1) / 2}\right), \quad \hbar \rightarrow 0\right)$ решение уравнения $(4.8)$, удовлетворяет начальному условию

$$
\left.\Phi\right|_{t=0}=\Psi_{0},
$$

где $\Psi_{0}(x, \hbar)$ определена в $(2.12)$, то функция $\Psi^{(N)}(x, t, \hbar)=\Phi^{(N)}\left(x, t, \hbar, \Psi_{0}\right)$ является асимптотическим ( с точностью $\left.O\left(\hbar^{(N+1) / 2}\right), \quad \hbar \rightarrow 0\right)$ решением задачи Коши для уравнения типа Хартри (2.9)-(2.12).

Для построения функции $\Phi^{(N)} \in \mathcal{P}_{\hbar}^{t}(Z(t, \hbar), S(t, \hbar)), N \geqslant 2$, воспользуемся методом построения локализованных асимптотик, развитым в работах [48], [59], [61], [62] для линейных квантово-механических уравнений, модифицировав его с учетом исходной постановки задачи (2.9)-(2.12) и структуры гамильтониана (4.7) для (линеаризованного) уравнения Хартри в траекторно-когерентном приближении. А именно точку локализации в $\mathbb{R}_{p, x}^{2 n}$ квазиклассически сосредоточенного решения уравнения (4.8) - "параметр" $Z(t, \hbar)$, входяший в определение класса $\mathcal{P}_{\hbar}^{t},-$ определим как естественную проекцию решения $y_{\Psi_{0}}^{N}(t, \hbar)$ системы Гамильтона-Эренфеста (3.2) на фазовое пространство, т.е. положим $Z(t, \hbar)=Z^{N}(t, \hbar)=\left(P^{N}(t, \hbar), X^{N}(t, \hbar)\right)$. Функцию $S(t, \hbar)$ (второй "параметр" класса $\left.\mathcal{P}_{\hbar}^{t}\right)$ определим как аналог классического действия вдоль этой $\left(z=Z^{N}(t, \hbar), t \in\right.$ $[0, T])$ фазовой траектории стандартной формулой. При этом будем исходить из классического гамильтониана, отвечаюшего не главному, а полному символу [1] $\mathcal{H}_{\varkappa}^{N}(z, t)$ квантового гамильтониана в (4.7). Этот символ с учетом оценок $\Delta_{\alpha}^{N}(t, \hbar)=O\left(\hbar^{|\alpha| / 2}\right)$ имеет вид

$$
\mathcal{H}_{\varkappa}^{N}(z, t)=\mathcal{H}(z, t)+\left.\tilde{\varkappa} \sum_{|\alpha|=0}^{N} \frac{1}{\alpha !} \frac{\partial^{|\alpha|}}{\partial w^{\alpha}} V(z, w, t)\right|_{w=Z^{N}(t, \hbar)} \Delta_{\alpha}^{N}(t, \hbar) .
$$

\footnotetext{
5) Тем самым мы подчеркиваем зависимость решения уравнения (4.8) (через коэффициенты оператора $\left.\hat{L}^{(N)}\left(t, \Psi_{0}\right)\right)$ от решения $y_{\Psi_{0}}^{N}(t, \hbar)$ уравнения Гамильтона-Эренфеста $(3.2)$ с начальными данными (3.3), индуцированными начальным условием $\Psi_{0}(2.12)$ в исходной постановке задачи (2.9)-(2.12) для уравнения типа Хартри.
} 
В результате получим

$$
S(t, \hbar)=S^{N}(t, \hbar)=\int_{0}^{t}\left[\left\langle P^{N}, \dot{X}^{N}\right\rangle(\tau, \hbar)-\mathcal{H}_{\varkappa}^{N}\left(Z^{N}(\tau, \hbar), \tau\right)\right] d \tau
$$

Обозначим

$$
S^{N}(x, t, \hbar)=S^{N}(t, \hbar)+\left\langle P^{N}(t, \hbar), x-X^{N}(t, \hbar)\right\rangle .
$$

Таким образом, для построения асимптотического $\bmod \hbar^{(N+1) / 2}, \quad \hbar \rightarrow 0$, решения уравнения (4.8)

$$
\Phi^{(N)}(x, t, \hbar)=e^{\frac{i}{\hbar} S^{N}(x, t, \hbar)} \varphi^{N}\left(\frac{\Delta x}{\sqrt{\hbar}}, t, \sqrt{\hbar}\right) \in \mathcal{P}_{\hbar}^{t}
$$

(здесь и далее зависимость решения $\Phi^{(N)}$ от $\Psi_{0}$ может быть опушена в целях сокрашения обозначений) достаточно найти амплитуду решения $\varphi^{N}$ с соответствуюшей точностью по $\hbar \rightarrow 0$ в виде разложения по степеням $\sqrt{\hbar}$,

$$
\varphi^{(N)}(x, t, \hbar)=\sum_{k=0}^{N-2} \hbar^{k / 2} \varphi_{k}^{N}\left(\frac{\Delta x}{\sqrt{\hbar}}, t\right)
$$

Соответственно для $\Phi^{(N)}(x, t, \hbar)$ имеем представление

$$
\Phi^{(N)}(x, t, \hbar)=\sum_{k=0}^{N-2} \hbar^{k / 2} \Phi_{k}^{(N)}(x, t, \hbar)
$$

где

$$
\Phi_{k}^{(N)}(x, t, \hbar)=e^{\frac{i}{\hbar} S^{N}(x, t, \hbar)} \varphi_{k}^{N}\left(\frac{\Delta x}{\sqrt{\hbar}}, t\right), \quad k=0, \ldots, N-2 .
$$

Далее, разложим операторы

$$
\mathcal{H}(\hat{z}, t) \quad \text { и }\left.\frac{\partial^{|\alpha|}}{\partial w^{\alpha}} V(\hat{z}, w, t)\right|_{w=z^{N}(t, \hbar)}
$$

в ряды Тейлора порядка $N$ по степеням оператора $\Delta \hat{z}=\hat{z}-Z^{N}(t, \hbar)$ с остаточными членами $\widehat{R}_{N+1}^{H}$ и $\widehat{R}_{N+1}^{V}$, соответственно, и представим оператор $-i \hbar \partial / \partial t$ в виде

$$
-i \hbar \partial_{t}=\hat{A}+\widehat{B}
$$

где

$$
\begin{aligned}
& \hat{A}=-i \hbar \partial_{t}-\dot{S}^{N}(t, \hbar)+\left\langle P^{N}(t, \hbar), \dot{X}^{N}(t, \hbar)\right\rangle+\left\langle\dot{Z}^{N}(t, \hbar), J \Delta \hat{z}\right\rangle, \\
& \widehat{B}=-\left\langle P^{N}(t, \hbar), \dot{X}^{N}(t, \hbar)\right\rangle+\dot{S}^{N}(t, \hbar)-\left\langle\dot{Z}^{N}(t, \hbar), J \Delta \hat{z}\right\rangle .
\end{aligned}
$$


Подставим полученные выражения в уравнение (4.8). С учетом оценок по $\hbar \rightarrow 0$ для операторов $\widehat{R}_{N+1}^{H}$ и $\widehat{R}_{N+1}^{V}$ на функциях класса $\mathcal{P}_{\hbar}^{t}$ (аналогичных оценке (4.3)) получим

$$
\left[-i \hbar \partial_{t}+\hat{\mathfrak{H}}_{0}\left(t, \Psi_{0}\right)+\hbar \hat{\mathfrak{H}}^{(N)}\left(t, \Psi_{0}\right)\right] \Phi=O\left(\hbar^{(N+1) / 2}\right) .
$$

В формуле (4.14) введены следуюшие обозначения:

$$
\begin{aligned}
\hat{\mathfrak{H}}_{0}\left(t, \Psi_{0}\right)= & -\dot{S}^{N}(t, \hbar)+\left\langle P^{N}(t, \hbar), \dot{X}^{N}(t, \hbar)\right\rangle+\left\langle\dot{Z}^{N}(t, \hbar), J \Delta \hat{z}\right\rangle+ \\
& +\frac{1}{2}\left\langle\Delta \hat{z}, \mathfrak{H}_{z z}\left(t, \Psi_{0}\right) \Delta \hat{z}\right\rangle,
\end{aligned}
$$

где

$$
\mathfrak{H}_{z z}\left(t, \Psi_{0}\right)=\left.\left[\mathcal{H}_{z z}(z, t)+\tilde{\varkappa} V_{z z}(z, w, t)\right]\right|_{z=w=Z^{N}(t, \hbar)},
$$

и

$$
\hat{\mathfrak{H}}^{(N)}\left(t, \Psi_{0}\right)=\sum_{k=1}^{N} \hbar^{k / 2} \hat{\mathfrak{H}}_{k}\left(t, \Psi_{0}\right)
$$

где

$$
\begin{aligned}
\hat{\mathfrak{H}}_{k}\left(t, \Psi_{0}\right)= & -\left\langle\dot{Z}_{(k+1)}(t), J \frac{\Delta \hat{z}}{\sqrt{\hbar}}\right\rangle+\left.\sum_{|\alpha|=k+2} \frac{1}{\alpha !} \frac{\partial^{|\alpha|} \mathcal{H}(z, t)}{\partial z^{\alpha}}\right|_{z=Z(t, \hbar, N)} \frac{\{\Delta \hat{z}\}^{\alpha}}{\hbar^{k+2}}+ \\
& +\left.\frac{\tilde{\varkappa}}{\hbar^{k+2}} \sum_{|\alpha+\beta|=k+2} \frac{1}{\alpha ! \beta !} \frac{\partial^{|\alpha+\beta|} V(z, w, t)}{\partial z^{\beta} \partial w^{\alpha}}\right|_{z=w=Z^{N}(t, \hbar)}\{\Delta \hat{z}\}^{\beta} \Delta_{\alpha}^{N}(t, \hbar) .
\end{aligned}
$$

Здесь функции $Z_{(k)}^{N}(t)$ - коэффициенты разложения проекции $Z^{N}(t, \hbar)$ решения $y^{(N)}(t, \hbar)$ системы Гамильтона-Эренфеста на фазовое пространство $\mathbb{R}^{2 n}$ в ряд регулярной теории возмушений по степеням $\sqrt{\hbar}$,

$$
Z^{N}(t, \hbar)=\sum_{k=0}^{N} \hbar^{k / 2} Z_{(k)}^{N}(t),
$$

причем в силу оценок $(2.4),(2.5)$ операторы $\hat{\mathfrak{H}}_{k}\left(t, \Psi_{0}\right), k=1, \ldots, N$, имеют по параметру $\hbar \rightarrow 0$ порядок $\widehat{O}(1)$ в смысле, определенном равенством (2.3). Отметим два важных технических момента, использованных при выводе приближенного уравнения (4.14). Во-первых, в силу оценки (2.4) для оператора $\hat{A}$ из (4.13) оператор $-i \hbar \partial / \partial t+\hat{\mathfrak{H}}_{0}\left(t, \Psi_{0}\right)$ имеет порядок $\widehat{O}(\hbar), \hbar \rightarrow 0$. Во-вторых, при подстановке в (4.8) оператора $\widehat{B}$ из (4.13), в котором

$$
\dot{Z}^{N}(t, \hbar)=\sum_{k=0}^{N} \hbar^{k / 2} \dot{Z}_{(k)}^{N}(t),
$$

слагаемые, пропорциональные $\dot{Z}_{(0)}^{N} \Delta \hat{z}$ и $\dot{Z}_{(1)}^{N} \Delta \hat{z}$, сокрашаются с соответствующими членами рядов Тейлора для $\mathcal{H}(\hat{z}, t)$ и

$$
\frac{\partial^{|\alpha|}}{\partial w^{\alpha}} V\left(\hat{z}, Z^{N}(t, \hbar), t\right) \text { при }|\alpha|=1
$$


в силу формул для нулевого $Z_{(0)}^{N}(t)$ и первого $Z_{(1)}^{N}(t)$ порядков приближения в решении задачи (3.2), (3.3) по теории возмушений.

Подставим теперь разложение (4.12) в (4.14) и приравняем нулю слагаемые при одинаковых степенях $\hbar^{1 / 2}$. В результате получим рекуррентную систему линейных ассоииированных уравнений для определения функций $\Phi_{k}^{(N)}(x, t, \hbar), k=0,1,2, \ldots, N-2$,

$$
\begin{aligned}
{\left[-i \hbar \frac{\partial}{\partial t}+\hat{\mathfrak{H}}_{0}\left(t, \Psi_{0}\right)\right] \Phi_{0}^{(N)} } & =0 \\
{\left[-i \hbar \frac{\partial}{\partial t}+\hat{\mathfrak{H}}_{0}\left(t, \Psi_{0}\right)\right] \Phi_{k}^{(N)}+\sum_{m=0}^{k-1} \hat{\mathfrak{H}}_{k-m}\left(t, \Psi_{0}\right) \Phi_{m}^{(N)} } & =0, \quad k=1,2, \ldots, N-2 .
\end{aligned}
$$

Уравнение (4.18) для главного члена асимптотического решения $\Phi_{0}^{(N)}(x, t, \hbar)$ естественно также назвать уравнением типа Хартри в траекторно-когерентном приближении $\bmod \hbar^{3 / 2}$. Это уравнение является уравнением Шредингера с гамильтонианом, квадратичным по операторам $\hat{p}$ и $\hat{x}$.

\section{TPАЕКТОРНО-КОГЕРЕНТНЫЕ СОСТОЯНИЯ УРАВНЕНИЯ ТИПА ХАРТРИ}

Решение линейного уравнения Шредингера с квадратичным гамильтонианом хорошо известно. Для наших целей удобно в качестве базиса решений уравнения (4.18) выбрать квазиклассические траекторно-когерентные состояния этого уравнения [59], [60]. В силу утверждения 4.1 такие состояния являются асимптотическими $\left(\bmod \hbar^{3 / 2}\right)$ решениями задачи (2.9)-(2.12), если функция $\Psi_{0}(x, \hbar)(2.12)$ совпадает с траекторно-когерентным состоянием в начальный момент времени. Эти решения мы также будем называть траекторно-когерентными состояниями уравнения типа Хартри (1.1). Приведем их явный вид, а также ряд свойств, которые ниже (в разделах 6,7 ) используются для решения задачи (2.9)-(2.12) с произвольным начальным условием из класса функций $\mathcal{P}_{\hbar}^{0}$

Пусть заданы натуральное число $N, N \geqslant 2$, и функция $\Psi_{0}(x, \hbar) \in \mathcal{P}_{\hbar}^{0}$. Обозначим через $B(t), C(t)$ комплексные $(n \times n)$-матрицы ${ }^{6)}$, удовлетворяющие системе в вариациях "с самодействием", отвечающей гамильтониану $\mathcal{H}_{\varkappa}^{0}(z, t)(4.10)$ - главному символу оператора $\hat{\mathfrak{H}}_{0}\left(t, \Psi_{0}\right)(4.15)$,

$$
\left(\begin{array}{c}
\dot{B} \\
\dot{C}
\end{array}\right)=J \mathfrak{H}_{z z}\left(t, \Psi_{0}\right)\left(\begin{array}{c}
B \\
C
\end{array}\right) \Longleftrightarrow\left\{\begin{array}{l}
\dot{B}=-\mathfrak{H}_{x p}\left(t, \Psi_{0}\right) C-\mathfrak{H}_{x x}\left(t, \Psi_{0}\right) B \\
\dot{C}=\mathfrak{H}_{p p}\left(t, \Psi_{0}\right) B+\mathfrak{H}_{p x}\left(t, \Psi_{0}\right) C
\end{array}\right.
$$

и начальным данным

$$
\left.B\right|_{t=0}=B_{0},\left.\quad C\right|_{t=0}=E_{n},
$$

6) Зависимость этих матриц от $N$ и $\Psi_{0}$ опускается. 
где $B_{0}$ - симметричная матрица с положительной мнимой частью

$$
B_{0}^{t}=B_{0}, \quad \operatorname{Im} B_{0}>0
$$

Здесь $(2 n \times 2 n)$-матрица $\mathfrak{H}_{z z}\left(t, \Psi_{0}\right)$ определена в $(4.15)$.

Как известно [83], линейная гамильтонова система (5.1) сохраняет стандартную симплектическую структуру $\omega=d p \wedge d x$ фазового пространства $\mathbb{R}_{p, x}^{2 n}$. Следовательно, кососкалярное произведение $\langle a, J b\rangle=\omega(a, b)$ любых двух комплексных решений $a(t), b(t) \in$ $\mathbb{C}_{w, z}^{2 n}$ системы (5.1) не зависит от времени (здесь через $\mathbb{C}_{w, z}^{2 n}$ обозначена комплексификация пространства $\mathbb{R}_{p, x}^{2 n}$ с комплексными координатами $\left.w \in \mathbb{C}^{n}, z \in \mathbb{C}^{n}, a=(w, z)\right)$. Отсюда и из условий (5.3) следуют равенства

$$
\begin{aligned}
C^{t}(t) B(t)-B^{t}(t) C(t) & =\left(\left\{a_{i}(t), a_{j}(t)\right\}\right)_{n \times n}=0 \\
\frac{1}{2 i}\left(C^{\dagger}(t) B(t)-B^{\dagger}(t) C(t)\right) & =\left(\frac{\left\{a_{i}(t), a_{j}^{*}(t)\right\}}{2 i}\right)_{n \times n}=\operatorname{Im} B_{0}>0,
\end{aligned}
$$

где $2 n$-мерный вектор $a_{i}(t)=\left(w_{i}(t), z_{i}(t)\right), i=1,2, \ldots, n$, составлен из вектор-столбцов $w_{i}(t)$ и $z_{i}(t)$ матриц $B(t)$ и $C(t)$, соответственно: $B(t)=\left(w_{1}(t), \ldots, w_{n}(t)\right), \quad C(t)=$ $\left(z_{1}(t), \ldots, z_{n}(t)\right)$. С помощью стандартных рассуждений (см., например, [62]) из (5.5) следует, что матрица $C(t)$ невырожденна, $\operatorname{det} C(t) \neq 0$, и мнимая часть матрицы

$$
Q(t)=B(t) C^{-1}(t)
$$

положительна при $t \in[0, T], T>0: \operatorname{Im} Q(t)>0$, причем в силу равенства (5.4) матрица $Q(t)$ симметрична, $Q^{t}(t)=Q(t)$.

Фиксируем теперь непрерывную ветвь корня из $\operatorname{det} C(t), t \in[0, T]$, например, считая, что $\operatorname{Arg} \sqrt{\operatorname{det} C(0)}=0$. Определим функцию

$$
\begin{aligned}
\Phi_{0}^{(N)}(x, t, \hbar)=\mid 0, & \left.t, \Psi_{0}\right\rangle=N_{0}(\hbar)(\operatorname{det} C(t))^{-1 / 2} \times \\
& \times \exp \left\{\frac{i}{\hbar}\left[S^{N}(t, \hbar)+\left\langle\frac{P^{N}(t, \hbar)}{\sqrt{\hbar}}, \frac{\Delta x}{\sqrt{\hbar}}\right\rangle+\frac{1}{2}\left\langle\frac{\Delta x}{\sqrt{\hbar}}, Q(t) \frac{\Delta x}{\sqrt{\hbar}}\right\rangle\right]\right\},
\end{aligned}
$$

где $\Delta x=x-X^{N}(t, \hbar),\left(P^{N}(t, \hbar), X^{N}(t, \hbar)\right)=Z^{N}(t, \hbar), t \in[0, T]$ - фазовая траектория в силу системы Гамильтона-Эренфеста порядка $N(3.2)$, функция $S^{N}(t, \hbar)$ определена в $(4.11)$ и $N_{0}(\hbar)=(\pi \hbar)^{-n / 4}\left(\operatorname{det} \operatorname{Im} B_{0}\right)^{1 / 4}$ - нормировочная постоянная, $\langle t, 0 \mid 0, t\rangle=1$.

ТеОрема 5.1. Пусть при заданных числе $N, \quad N \geqslant 2$, и функиии $\Psi_{0}(x, \hbar) \in \mathcal{P}_{0}^{\hbar}$ на интервале [0,T], T>0, существует решение задачи Коши (3.2), (3.3), гладко зависящее от параметра $\sqrt{\hbar}, \hbar \in(0,1]$. Тогда при $t \in[0, T]$ функиия $\Phi_{0}^{(N)}(x, t, \hbar)$ (5.7) является точным решением задачи Коши для уравнения (4.18) с начальным условием

$$
\left.\Phi_{0}^{(N)}\right|_{t=0}=N_{0}(\hbar) \exp \left\{\frac{i}{\hbar}\left[\left\langle p_{0}, x-x_{0}\right\rangle+\frac{1}{2}\left\langle x-x_{0}, B_{0}\left(x-x_{0}\right)\right\rangle\right]\right\},
$$

где $\left(p_{0}, x_{0}\right) \in \mathbb{R}_{p, x}^{2 n}$ и комплексная $(n \times n)$-матрица В $B_{0}$ удовлетворяет условиям (5.3). 
ДокаЗАтельство. Решение линейного уравнения (4.18) в классе функций $\mathcal{P}_{\hbar}^{t}\left(S^{N}(t, \hbar), Z^{N}(t, \hbar)\right)$ ишем в форме гауссова пакета

$$
\Phi(x, t, \hbar)=\exp \left\{\frac{i}{\hbar}\left[S^{N}(t, \hbar)+\left\langle P^{N}(t, \hbar), \Delta x\right\rangle\right]\right\} \exp \left\{\frac{i}{\hbar} \frac{\langle\Delta x, Q(t) \Delta x\rangle}{2}\right\} \varphi(t),
$$

где комплексная $(n \times n)$-матрица $Q(t)$ симметрична и $\operatorname{Im} Q(t)>0$. Подставив $(5.9)$ в (4.18) и приравняв нулю коэффициенты при степенях оператора $\Delta x^{k}, k=0,2$, получим линейное уравнение на функцию $\varphi(t)$ и матричное уравнение Риккати на матрицу $Q(t)$, соответственно,

$$
\begin{gathered}
\dot{\varphi}+\frac{1}{2} \operatorname{Sp}\left[\mathfrak{H}_{p x}\left(t, \Psi_{0}\right)+\mathfrak{H}_{p p}\left(t, \Psi_{0}\right) Q(t)\right] \varphi=0 \\
\dot{Q}+\mathfrak{H}_{x x}\left(t, \Psi_{0}\right)+Q(t) \mathfrak{H}_{p x}\left(t, \Psi_{0}\right)+\mathfrak{H}_{x p}\left(t, \Psi_{0}\right) Q(t)+Q(t) \mathfrak{H}_{p p}\left(t, \Psi_{0}\right) Q(t)=0 .
\end{gathered}
$$

Стандартная замена $Q(t)=B(t) C^{-1}(t)$ (см., например, [84]) при условии, что $Q(0)=$ $B_{0}$, где $B_{0}$ удовлетворяет условиям (5.3), сводит задачу построения требуемого комплексного решения уравнения (5.11) к задаче (5.1), (5.2). В силу второго уравнения системы (5.1) имеем

$$
\dot{C}=\left[\mathfrak{H}_{p x}\left(t, \Psi_{0}\right)+\mathfrak{H}_{p p}\left(t, \Psi_{0}\right) Q(t)\right] C,
$$

где $Q(t)$ - решение уравнения (5.11). Отсюда в силу леммы Лиувилля найдем, что

$$
\operatorname{det} C(t)=\exp \int_{0}^{t} \operatorname{Sp}\left[\mathfrak{H}_{p x}\left(\tau, \Psi_{0}\right)+\mathfrak{H}_{p p}\left(\tau, \Psi_{0}\right) Q(\tau)\right] d \tau
$$

и, следовательно, из уравнения $(5.10)$ имеем $\varphi(t)=(\operatorname{det} C(t))^{-1 / 2}$.

Построим теперь фоковский базис решений уравнения (4.18). Для этого будем искать линейные по операторам $\Delta \hat{z}$ операторы симметрии $\hat{a}\left(t, \Psi_{0}\right)$ этого уравнения в виде $\hat{a}\left(t, \Psi_{0}\right)=N_{a}\left\langle b\left(t, \Psi_{0}\right), \Delta \hat{z}\right\rangle$, где $N_{a}$ - постоянная, $b(t)=b\left(t, \Psi_{0}\right)$ - комплексный $2 n$-вектор, подлежаший определению. Из уравнения

$$
-i \hbar \frac{\partial \hat{a}(t)}{\partial t}+\left[\hat{\mathfrak{H}}_{0}\left(t, \Psi_{0}\right), \hat{a}(t)\right]=0
$$

определяющего оператор $\hat{a}(t)$, с учетом явного вида $\hat{\mathfrak{H}}_{0}\left(t, \Psi_{0}\right)(4.15)$ получим

$$
\begin{aligned}
& -i \hbar\langle\dot{b}(t), \Delta \hat{z}\rangle+i \hbar\left\langle b(t), \dot{Z}^{N}(t, \hbar)\right\rangle+\left[-\dot{S}^{N}(t, \hbar)+\left\langle P^{N}(t, \hbar), \dot{X}^{N}(t, \hbar)\right\rangle+\right. \\
& \left.+\left\langle\dot{Z}^{N}(t, \hbar), J \Delta \hat{z}\right\rangle+\frac{1}{2}\left\langle\Delta z, \mathfrak{H}_{z z}\left(t, \Psi_{0}\right) \Delta \hat{z}\right\rangle,\langle b(t), \Delta \hat{z}\rangle\right]=0
\end{aligned}
$$

Отсюда в силу коммутационных соотношений $\left[\Delta \hat{z}_{j}, \Delta \hat{z}_{k}\right]=i \hbar J_{j k}, j, k=1, \ldots, 2 n$, найдем

$$
-i \hbar\langle\dot{b}(t), \Delta \hat{z}\rangle+i \hbar\left\langle\Delta \hat{z}, \mathfrak{H}_{z z}(t) J b(t)\right\rangle=0
$$

5 Теоретическая и математическая физика, т. 130, № 3, 2002 г. 
и, следовательно, $\dot{b}=\mathfrak{H}_{z z}\left(t, \Psi_{0}\right) J b$. Обозначим $b(t)=-J a(t)$. Тогда для определения вектора $a(t)$ получим уравнение $\dot{a}=J \mathfrak{H}_{z z}\left(t, \Psi_{0}\right) a$. Таким образом, оператор

$$
\hat{a}(t)=\hat{a}\left(t, \Psi_{0}\right)=N_{a}\langle b(t), \Delta \hat{z}\rangle=N_{a}\langle a(t), J \Delta \hat{z}\rangle
$$

является оператором симметрии для уравнения (4.18), если вектор $a(t)=a\left(t, \Psi_{0}\right)$ является решением системы в вариациях (5.1).

Пусть $\hat{a}(t), \hat{b}(t)$ - операторы симметрии, отвечаюшие двум решениям системы в вариациях $a(t)$ и $b(t)$, соответственно. Тогда нетрудно проверить, что

$$
[\hat{a}(t), \hat{b}(t)]=i \hbar N_{a} N_{b}\{a(t), b(t)\}=i \hbar N_{a} N_{b}\{a(0), b(0)\},
$$

причем последнее равенство является следствием свойства гамильтоновости системы (5.1).

Пусть теперь матрица $B_{0}$ в (5.2) диагональна, $B_{0}=\operatorname{diag}\left(b_{1}, \ldots, b_{n}\right)$, где $b_{j} \in \mathbb{C}$, $\operatorname{Im} b_{j}>0, j=1,2, \ldots, n$. Тогда вектор-столбшы $a_{j}(t)=\left(w_{j}(t), z_{j}(t)\right), j=1,2, \ldots, n$, $(2 n \times 2 n)$-матришы $\left(\begin{array}{c}B(t) \\ C(t)\end{array}\right)$ представляют собой решения задачи $(5.1),(5.2)$ и векторы $a_{j}^{*}(t), j=1,2, \ldots, n$, образуют базис решений системы в вариациях. По формуле (5.12) сопоставим векторам $a_{j}^{*}(t)$ операторы "рождения" $\hat{a}_{j}^{+}(t)$, а векторам $a_{j}(t)$ - операторы "уничтожения" $\hat{a}_{j}(t)$, положив $N_{j}=\left(\hbar \operatorname{Im} b_{j}\right)^{-1 / 2}$. Тогда в силу формул (5.13) для операторов $\hat{a}_{j}^{+}(t), \hat{a}_{j}(t), j=1,2, \ldots, n$, справедливы канонические коммутационные соотношения

$$
\left[\hat{a}_{j}(t), \hat{a}_{k}(t)\right]=\left[\hat{a}_{j}^{+}(t), \hat{a}_{k}^{+}(t)\right]=0, \quad\left[\hat{a}_{j}(t), \hat{a}_{k}^{+}(t)\right]=\delta_{j k}, \quad j, k=1,2, \ldots, n .
$$

УТвеРЖДЕнИЕ 5.1. Пусть в (5.2) $B_{0}=\operatorname{diag}\left(b_{1}, \ldots, b_{n}\right), \quad b_{j} \in \mathbb{C}, \quad \operatorname{Im} b_{j}>0$, $j=1, \ldots, n$. Тогда функция $\Phi_{0}^{(N)}(x, t, \hbar)=\left|0, t, \Psi_{0}\right\rangle$ (5.7) является "вакуумным" траекторно-когерентным состоянием,

$$
\hat{a}_{j}(t)\left|0, t, \Psi_{0}\right\rangle=0, \quad j=1,2, \ldots, n .
$$

ДокАЗАТЕльСТво. Действуя оператором “уничтожения" $\hat{a}_{j}(t)$ на функцию $|0, t\rangle$, получим, что

$$
\hat{a}_{j}(0, t)|0, t\rangle=\left(\left\langle z_{j}(t), Q(t) \Delta x\right\rangle-\left\langle w_{j}(t), \Delta x\right\rangle\right)|0, t\rangle .
$$

Отсюда немедленно следует (5.15), поскольку в силу определения и свойств матришы $Q(t)$ имеем

$$
Q(t) z_{j}(t)=B(t) C^{-1}(t) z_{j}(t)=w_{j}(t)
$$


При заданных $N \geqslant 2$ и $\Psi_{0} \in \mathcal{P}_{\hbar}^{0}$ определим теперь счетный набор состояний $\left|\nu, t, \Psi_{0}\right\rangle$ (точных решений уравнения (4.18)) как результат действия операторов “рождения" на “вакуумное" состояние $\left|0, t, \Psi_{0}\right\rangle(5.7)$,

$$
\Phi_{\nu}^{(N)}(x, t, \hbar)=\left|\nu, t, \Psi_{0}\right\rangle=\frac{1}{\nu !}\left(\hat{a}^{+}\left(t, \Psi_{0}\right)\right)^{\nu}\left|0, t, \Psi_{0}\right\rangle=\prod_{k=1}^{n} \frac{1}{\nu_{k} !}\left(\hat{a}_{k}^{+}\left(t, \Psi_{0}\right)\right)^{\nu_{k}}\left|0, t, \Psi_{0}\right\rangle
$$

Функции $\Phi_{\nu}^{(N)}(x, t, \hbar), \nu \in \mathbb{Z}_{+}^{n}$, образуют фоковский базис решений (линейного) уравнения (4.18). Действительно, используя формулы (5.15), (5.14), с помощью стандартных вычислений легко проверить ортонормированность этого набора функций

$$
\left\langle\Phi_{\nu}^{(N)}, \Phi_{\nu^{\prime}}^{(N)}\right\rangle=\delta_{\nu \nu^{\prime}}, \quad \nu, \nu^{\prime} \in \mathbb{Z}_{+}^{n}
$$

Доказательство полноты следует, например, из результатов [85]. Таким образом, отсюда и из утверждения 4.1 вытекает следующая теорема.

ТЕОРема 5.2. Пусть символь операторов $\widehat{\mathcal{H}}(t)$ и $\widehat{V}(t, \Psi)$ в (1.1) удовлетворяют условиям предположения 2.1 и пусть выполнены условия теоремы 5.1. Тогда для любого $\nu \in \mathbb{Z}_{+}^{n}$ функиия $\Psi_{\nu}^{(N)}(x, t, \hbar)$ является асимптотическим (с точностью $\left.O\left(\hbar^{3 / 2}\right), \hbar \rightarrow 0\right)$ решением уравнения типа Хартри (1.1) с начальным условием

$$
\begin{aligned}
\left.\Psi\right|_{t=0} & =\Psi_{0}(x, \hbar)=\frac{1}{\nu !} \hat{a}^{+}(0)^{\nu} \Phi_{0}^{(N)}(x, \hbar)= \\
& =e^{\frac{i}{\hbar}\left\langle p_{0}, x-x_{0}\right\rangle} N_{0}(\hbar) \prod_{j=1}^{n} \frac{e^{\frac{i}{\hbar} b_{j} \frac{\left(x_{j}-x_{j_{0}}\right)^{2}}{2}}}{\sqrt{2^{\nu_{j}} \nu_{j} !}} H_{\nu_{j}}\left(\frac{x_{j}-x_{j_{0}}}{\sqrt{\hbar}} \sqrt{\operatorname{Im} b_{j}}\right),
\end{aligned}
$$

где функиия $\Phi_{0}^{(N)}(x, \hbar)$ определена формулой $(5.8), \quad H_{\nu_{j}}(\xi), \quad \xi \in \mathbb{R},-$ полином Эрмита степени $\nu_{j} \in \mathbb{Z}_{+}, \quad N_{0}(\hbar)=(\pi \hbar)^{-n / 4}\left(\prod_{j=1}^{n} \operatorname{Im} b_{j}\right)^{1 / 4}$.

\section{6. КВАЗИКЛАССИЧЕСКИ СОСРЕДОТОЧЕННЫЕ РЕШЕНИЯ УРАВНЕНИЯ ТИПА ХАРТРИ (ГЛАВНЫЙ ЧЛЕН АСИМПТОТИКИ)}

Асимптотические решения задачи Коши (2.9)-(2.12) (где $\Psi_{0}$ имеет вид $(5.17)$ ), построенные в предыдушем разделе, являются частным случаем квазиклассически сосредоточенных $\left(\bmod \hbar^{3 / 2}\right)$ решений уравнения $(1.1)$. В случае произвольных начальных условий $\Psi_{0}(x, \hbar)(2.12)$, принадлежащих классу $\mathcal{P}_{\hbar}^{0}$, главный член асимптотики $\Psi_{0}^{(N)}(x, t, \hbar)$ уравнения (1.1) определяется разложением в ряд по фоковскому базису $\left|\nu, t, \Psi_{0}\right\rangle(5.16), \nu \in \mathbb{Z}_{+}^{n}$, решений уравнения (4.18),

$$
\Psi_{0}^{(N)}(x, t, \hbar)=\sum_{|\nu|=0}^{\infty} C_{\nu}\left|\nu, t, \Psi_{0}\right\rangle, \quad C_{\nu}=\left\langle\Psi_{0}, 0, \nu \mid \Psi_{0}\right\rangle
$$


В дальнейшем более удобным для нас является представление решения $\Psi_{0}^{(N)}(x, t, \hbar)$ через свертку начального условия $\Psi_{0}$ с функцией Грина $G_{0}^{(N)}\left(x, y, t, s, \Psi_{0}\right)$ уравнения (4.18). Функция Грина для квадратичных квантовых систем известна (см., например, $[71],[86],[87])$. Для полноты изложения приведем ее явный вид в форме, позволяющей явно показать нетривиальную зависимость оператора эволюции ассоциированного линейного уравнения (4.18) от начальных условий для исходного уравнения типа Хартри.

По определению функции Грина $G_{0}^{(N)}$ для уравнения (4.18) имеем

$$
\begin{gathered}
{\left[-i \hbar \partial_{t}+\hat{\mathfrak{H}}_{0}\left(t, \Psi_{0}\right)\right] G_{0}^{(N)}\left(x, y, t, s, \Psi_{0}\right)=0, \quad 0 \leqslant s \leqslant t,} \\
\lim _{t \rightarrow s} G_{0}^{(N)}\left(x, y, t, s, \Psi_{0}\right)=\delta(x-y),
\end{gathered}
$$

где оператор $\hat{\mathfrak{H}}_{0}$ определен в $(4.15)$. Обозначим через $\lambda_{k}\left(t, \Psi_{0}\right), k=1,2,3,4,(n \times n)$-матрицы, являюшиеся блоками фундаментальной матрицы системы в вариациях (5.1),

$$
\Phi\left(t, \Psi_{0}\right)=\left(\begin{array}{cc}
\lambda_{4}^{t}\left(t, \Psi_{0}\right) & \lambda_{2}^{t}\left(t, \Psi_{0}\right) \\
\lambda_{3}^{t}\left(t, \Psi_{0}\right) & \lambda_{1}^{t}\left(t, \Psi_{0}\right)
\end{array}\right), \quad \Phi\left(0, \Psi_{0}\right)=E_{2 n \times 2 n} .
$$

Пусть выполнены следующие условия:

$$
\operatorname{det} \mathfrak{H}_{p p}\left(t, \Psi_{0}\right) \neq 0, \quad \operatorname{det} \lambda_{3}(t-s) \neq 0, \quad s, t \in[0, T] .
$$

Тогда функция Грина $G_{0}^{(N)}\left(x, y, t, s, \Psi_{0}\right)$ имеет вид [61], [62]

$$
\begin{aligned}
G_{0}^{(N)}\left(x, y, t, s, \Psi_{0}\right)= & \frac{1}{\sqrt{\operatorname{det}\left(-i 2 \pi \hbar \lambda_{3}(\Delta t)\right)}} \exp \left\{\frac { i } { \hbar } \left[S^{N}(t, \hbar)-S^{N}(s, \hbar)+\right.\right. \\
& +\left\langle P^{N}(t, \hbar), \Delta x\right\rangle-\left\langle p_{0}, y-x_{0}\right\rangle- \\
& -\frac{1}{2}\left\langle y-x_{0}, \lambda_{1}(\Delta t) \lambda_{3}^{-1}(\Delta t)\left(y-x_{0}\right)\right\rangle-\frac{1}{2}\left\langle\Delta x, \lambda_{3}^{-1}(\Delta t)\left(y-x_{0}\right)\right\rangle- \\
& \left.\left.-\frac{1}{2}\left\langle\Delta x, \lambda_{3}^{-1}(\Delta t) \lambda_{4}(\Delta t) \Delta x\right\rangle\right]\right\} .
\end{aligned}
$$

Здесь $\Delta t=t-s,\left(P^{N}(t, \hbar), X^{N}(t, \hbar)\right)$ - проекция решения системы Гамильтона-Эренфеста порядка $N$ на пространство $\mathbb{R}_{p, x}^{2 n}$.

ЗАмЕЧАниЕ 6.1. Если условия (6.3) не выполняются, то решение задачи (6.1) в несколько иной форме, чем (6.4), можно найти, например, в работах [86], [87].

Таким образом, отсюда и из утверждения 4.1 следует теорема.

ТЕОРема 6.1. Пусть символы операторов $\widehat{\mathcal{H}}(t)$ и $\widehat{V}(t, \Psi)$ в (1.1) удовлетворяют условиям предположсения 2.1 и пусть выполнены условия теоремы 5.1 и условия (6.3). Тогда функиия

$$
\Psi_{0}^{(N)}(x, t, \hbar)=\widehat{U}_{0}^{(N)}\left(t, 0, \Psi_{0}\right) \Psi_{0}, \quad t \in[0, T],
$$

где $\widehat{U}_{0}^{(N)}\left(t, 0, \Psi_{0}\right)$ - оператор эволючии ассоциированного уравнения Шредингера нулевого порядка (4.18) с ядром $G_{0}^{(N)}\left(x, y, t, 0, \Psi_{0}\right)(6.4)$, является асимптотическим (с точностью $\left.O\left(\hbar^{3 / 2}\right), \hbar \rightarrow 0\right)$ решением уравнения типа Хартри (1.1) с начальным условием

$$
\left.\Psi\right|_{t=0}=e^{\frac{i}{\hbar}\left\langle p_{0}, x-x_{0}\right\rangle} \varphi_{0}\left(\frac{x-x_{0}}{\sqrt{\hbar}}\right), \quad \varphi_{0}(\xi) \in \mathbb{S}\left(\mathbb{R}^{n}\right) .
$$




\section{7. КВАЗИКЛАССИЧЕСКИ СОСРЕДОТОЧЕННЫЕ РЕШЕНИЯ УРАВНЕНИЯ ТИПА ХАРТРИ (ВЫСШИЕ ПРИБЛИЖЕНИЯ)}

Построим квазиклассически сосредоточенные решения $\left(\bmod \hbar^{(N+1) / 2}, \hbar \rightarrow 0, N \geqslant 2\right)$ задачи Коши для уравнения (1.1) с произвольным начальным условием $\Psi_{0}(x, \hbar) \in \mathcal{P}_{\hbar}^{0}$, которое с соответствуюшей точностью по $\hbar \rightarrow 0$ имеет вид

$$
\left.\Psi\right|_{t=0}=\Psi_{0}(x, \hbar)=\sum_{k=0}^{N-2} \hbar^{k / 2} \Phi_{k}(x, \hbar)+O\left(\hbar^{N / 2}\right),
$$

где

$$
\Phi_{k}(x, \hbar)=e^{\frac{i}{\hbar}\left\langle p_{0}, x-x_{0}\right\rangle} \varphi_{k}\left(\frac{x-x_{0}}{\sqrt{\hbar}}\right), \quad \varphi_{k}(\xi) \in S\left(\mathbb{R}_{x}^{n}\right), \quad k=0,1, \ldots, N-2 .
$$

Тогда для рекуррентной системы ассоциированных линейных уравнений (4.18), (4.19) имеем задачу Коши со следуюшими начальными данными:

$$
\left.\Phi_{k}^{(N)}\right|_{t=0}=\Phi_{k}(x, 0, \hbar), \quad k=0,1, \ldots, N-2 .
$$

Решение этой задачи нетрудно построить в виде разложения по полному ортонормированному набору фоковских функций $\left|\nu, t, \Psi_{0}\right\rangle(5.16)$, в котором “параметр" $\Psi_{0}$ определяется главным членом разложения начального условия (7.1),

$$
\Psi_{0}=\Phi_{0}(x, \hbar) .
$$

В результате получим

$$
\begin{aligned}
\Phi_{0}^{(N)}(x, t, \hbar)= & \sum_{|\nu|=0}^{\infty}\left|\nu, t, \Psi_{0}\right\rangle\left\langle\Psi_{0}, 0, \nu \mid \Phi_{0}(x, \hbar)\right\rangle, \\
\Phi_{1}^{(N)}(x, t, \hbar)= & \sum_{|\nu|=0}^{\infty}\left|\nu, t, \Psi_{0}\right\rangle\left\langle\Psi_{0}, 0, \nu \mid \Phi_{1}(x, \hbar)\right\rangle- \\
& -\frac{i}{\hbar} \sum_{|\nu|=0}^{\infty}\left|\nu, t, \Psi_{0}\right\rangle \int_{0}^{t} d \tau\left\langle\Psi_{0}, \tau, \nu \mid \hat{\mathfrak{H}}_{1}\left(t, \Psi_{0}\right) \Phi_{0}^{(N)}(x, \tau, \hbar)\right\rangle, \\
\Phi_{2}^{(N)}(x, t, \hbar)= & \sum_{|\nu|=0}^{\infty}\left|\nu, t, \Psi_{0}\right\rangle\left\langle\Psi_{0}, 0, \nu \mid \Phi_{2}(x, \hbar)\right\rangle- \\
& -\frac{i}{\hbar} \sum_{|\nu|=0}^{\infty}\left|\nu, t, \Psi_{0}\right\rangle \int_{0}^{t} d \tau\left\langle\Psi_{0}, \tau, \nu \mid \hat{\mathfrak{H}}_{1}\left(t, \Psi_{0}\right) \Phi_{1}^{(N)}(x, \tau, \hbar)\right\rangle- \\
& -\frac{i}{\hbar} \sum_{|\nu|=0}^{\infty}\left|\nu, t, \Psi_{0}\right\rangle \int_{0}^{t} d \tau\left\langle\Psi_{0}, \tau, \nu \mid \hat{\mathfrak{H}}_{2}\left(t, \Psi_{0}\right) \Phi_{0}^{(N)}(x, \tau, \hbar)\right\rangle,
\end{aligned}
$$


Обозначим через $\widehat{\mathcal{F}}^{(N)}\left(t, \Psi_{0}\right)$ оператор, определенный соотношением

$$
\widehat{\mathcal{F}}^{(N)}\left(t, \Psi_{0}\right) \Phi(x, t)=\int_{0}^{t} d \tau \widehat{U}_{0}^{(N)}\left(t, \tau, \Psi_{0}\right) \hat{\mathfrak{H}}^{(N)}\left(\tau, \Psi_{0}\right) \Phi(x, \tau)
$$

где $\widehat{U}_{0}^{(N)}\left(t, \tau, \Psi_{0}\right)$ - оператор эволюции ассоциированного уравнения Шредингера (4.18) при $\Psi_{0}=\Phi_{0}$ и оператор $\hat{\mathfrak{H}}^{(N)}\left(t, \Psi_{0}\right)=\sum_{k=1}^{N} \hbar^{k / 2} \hat{\mathfrak{H}}_{k}\left(t, \Psi_{0}\right)$ определен в $(4.16)$. Тогда из формул (7.1)-(7.6) и утверждения 4.1 вытекает следуюшая теорема.

ТЕОРЕма 7.1. Пусть выполнены условия теоремы 6.1. Тогда функиия

$$
\Psi^{(N)}(x, t, \hbar)=\sum_{k=0}^{N-2} \frac{1}{k !}\left[-\frac{i}{\hbar} \widehat{\mathcal{F}}^{(N)}\left(t, \Psi_{0}\right)\right]^{k} \widehat{U}_{0}^{(N)}\left(t, 0, \Psi_{0}\right) \Psi_{0}(x, \hbar)
$$

где $N \geqslant 2$, при $t \in[0, T]$ является асимптотическим с точностью $O\left(\hbar^{(N+1) / 2}\right)$, $\hbar \rightarrow 0$, решением уравнения (1.1) и удовлетворяет начальному условию (7.1), (7.2).

\section{8. ФУНКЦИЯ ГРИНА И НЕЛИНЕЙНЫЙ ПРИНЦИП СУПЕРПОЗИЦИИ}

Покажем, что в классе траекторно-сосредоточенных функций для уравнения (1.1) с любой заданной точностью по $\hbar^{1 / 2}, \hbar \rightarrow 0$, можно построить ядро оператора эволюции - функцию Грина соответствующей задачи Коши. Явный вид квазиклассических асимптотик $\Psi^{(N)}(x, t, \hbar)(7.7)$ позволяет получить представление для этой функции Грина $G^{(N)}\left(x, y, t, s, \Psi_{0}\right)$ (справедливое на конечном промежутке $t \in[0, T]$, где выполняются условия (6.3)). Действительно, согласно (7.7) для любой функции $\Psi_{0}(x, \hbar) \in \mathcal{P}_{\hbar}^{0}$ решение задачи Коши с начальным условием

$$
\left.\Phi(x, t, \hbar)\right|_{t=0}=\Psi_{0}(x, \hbar)
$$

для линейного ассоциированного уравнения Шредингера (4.8) порядка $N$ имеет вид

$$
\begin{aligned}
\Phi^{(N)}\left(x, t, \hbar, \Psi_{0}\right) & =\widehat{R}^{(N)}\left(t, \Psi_{0}\right) \int_{\mathbb{R}^{n}} d y G_{0}^{(N)}\left(x, y, t, 0, \Psi_{0}\right) \varphi(y, \hbar)+O\left(\hbar^{(N+1) / 2}\right)= \\
& =\int_{\mathbb{R}^{n}} d y G^{(N)}\left(x, y, t, 0, \Psi_{0}\right) \varphi(y, \hbar)+O\left(\hbar^{(N+1) / 2}\right),
\end{aligned}
$$

где через $\widehat{R}^{(N)}\left(t, \Psi_{0}\right)$ обозначен оператор

$$
\widehat{R}^{(N)}\left(t, \Psi_{0}\right)=\sum_{k=0}^{N-2} \frac{1}{k !}\left[-\frac{i}{\hbar} \widehat{\mathcal{F}}^{(N)}\left(t, \Psi_{0}\right)\right]^{k}
$$


Напомним, что функция $G_{0}^{(N)}\left(x, y, t, s, \Psi_{0}\right)$ и оператор $\widehat{\mathcal{F}}^{(N)}\left(t, \Psi_{0}\right)$ определены формулами (6.4) и (7.6), соответственно. Следовательно, для ядра интегрального оператора $G^{(N)}$ в $(8.2)$ имеем

$$
G^{(N)}\left(x, y, t, 0, \Psi_{0}\right)=\widehat{R}^{(N)}\left(t, \Psi_{0}\right) G_{0}^{(N)}\left(x, y, t, 0, \Psi_{0}\right) .
$$

Поскольку $\widehat{R}^{(N)}\left(0, \Psi_{0}\right)=1$, то для произвольного начального момента времени $s, 0<$ $s<t$, отсюда следует представление функции Грина задачи Коши (4.8), (8.1) с $s \neq 0$,

$$
G^{(N)}\left(x, y, t, s,, \Psi_{0}\right)=\widehat{R}^{(N)}\left(t, \Psi_{0}\right) G_{0}^{(N)}\left(x, y, t, s, \Psi_{0}\right)\left(\widehat{R}^{(N)}\left(s, \Psi_{0}\right)\right)^{+} .
$$

Нетрудно проверить для функций $G^{(N)}\left(x, y, t, s, \Psi_{0}\right)$ следующее правило композиции:

$$
\int_{\mathbb{R}^{n}} d u G^{(N)}\left(x, u, t, \tau, \Psi_{0}\right) G^{(N)}\left(u, y, \tau, s, \Psi_{0}\right)=G^{(N)}\left(x, y, t, s, \Psi_{0}\right)+O\left(\hbar^{(N+1) / 2}\right) .
$$

Обозначим через $\widehat{U}^{(N)}\left(t, 0, \Psi_{0}\right)$ приближенный оператор эволюции линейного уравнения (4.18),

$$
\widehat{U}^{(N)}\left(t, 0, \Psi_{0}\right) \varphi(x, \hbar)=\int_{\mathbb{R}^{n}} d y G^{(N)}\left(x, y, t, 0, \Psi_{0}\right) \varphi(y, \hbar), \quad \varphi(x, \hbar) \in L_{2}\left(\mathbb{R}^{n}\right) .
$$

В силу (8.4) его можно записать в виде $T$-упорядоченного дайсоновского разложения

$$
\widehat{U}^{(N)}\left(t, 0, \Psi_{0}\right)=\sum_{k=0}^{N-2}\left(-\frac{i}{\hbar}\right)^{k} \int_{\Delta_{k}^{>}} d^{k} \tau \hat{\mathfrak{H}}_{1}^{(N)}\left(\tau_{1}, t, \Psi_{0}\right) \ldots \hat{\mathfrak{H}}_{1}^{(N)}\left(\tau_{k}, t, \Psi_{0}\right) \widehat{U}_{0}^{(N)}\left(t, 0, \Psi_{0}\right) .
$$

Здесь использованы следующие обозначения: область интегрирования - открытый гипертреугольник

$$
\Delta_{k}^{>} \equiv\left\{\tau \in[0, t]^{k} ; \quad t>\tau_{1}>\tau_{2}>\cdots>\tau_{N}>s\right\},
$$

оператор $\hat{\mathfrak{H}}_{1}^{(N)}\left(\tau, t, \Psi_{0}\right)$ - оператор “возмушения" $\hat{\mathfrak{H}}^{(N)}\left(t, \Psi_{0}\right)(4.16)$ в уравнении (4.18),

$$
\hat{\mathfrak{H}}_{1}^{(N)}\left(\tau, t, \Psi_{0}\right)=\widehat{U}_{0}^{(N)}\left(t, \tau, \Psi_{0}\right) \hat{\mathfrak{H}}^{(N)}\left(\tau, \Psi_{0}\right)\left(\widehat{U}_{0}^{(N)}\left(\tau, t, \Psi_{0}\right)\right)^{+},
$$

а $\widehat{U}_{0}^{(N)}\left(t, s, \Psi_{0}\right)$ - оператор эволюции ассоциированного линейного уравнения Шредингера нулевого порядка (4.18) с ядром $G_{0}^{(N)}\left(x, y, t, s, \Psi_{0}\right)(6.4)$.

Из соотношения (8.2) и утверждения 4.1 вытекает, что действие оператора (8.5) на функцию $\Psi_{0}(x, \hbar)$ определяет асимптотическое по $\bmod \hbar^{(N+1) / 2}$ решение задачи Коши (2.9)-(2.12) для уравнения типа Хартри в виде

$$
\Psi^{(N)}(x, t, \hbar)=\widehat{U}^{(N)}\left(t, 0, \Psi_{0}\right) \Psi_{0}(x, \hbar), \quad \Psi_{0}(x, \hbar) \in \mathcal{P}_{\hbar}^{0} .
$$

Следовательно, оператор (8.5) является приближенным (с точностью $O\left(\hbar^{(N+1) / 2}\right)$, $\hbar \rightarrow 0$ ) оператором эволюции для уравнения (1.1) в классе траекторно-сосредоточенных функций.

Из представления (8.7) для построенных асимптотических решений непосредственно следует (см. также [88]-[90]) теорема. 
ТЕорема 8.1 (нелинейный принцип суперпозиции). Пусть $\Psi_{j}\left(x, t, \hbar, y_{j}^{(N)}(t, \hbar)\right)$ асимптотическое с точностью $O\left(\hbar^{(N+1) / 2}\right)$ решение задачи Кочи для уравнения (1.1) с начальным условием $\Psi_{0 j}(x, \hbar) \in \mathcal{P}_{0}^{\hbar}, j=1,2$. Тогда решение задачи Кочи для әтого уравнения с начальным условием

$$
\Psi_{03}(x, \hbar)=c_{1} \Psi_{01}(x, \hbar)+c_{2} \Psi_{02}(x, \hbar), \quad c_{1}, c_{2}=\text { const },
$$

имеет вид

$$
\begin{aligned}
\Psi_{3}\left(x, t, \hbar, y_{3}^{(N)}(t, \hbar)\right) & =\widehat{U}^{(N)}\left(t, 0, \Psi_{03}\right) \Psi_{03}(x)= \\
& =c_{1} \widehat{U}^{(N)}\left(t, 0, \Psi_{03}\right) \Psi_{01}(x)+c_{2} \widehat{U}^{(N)}\left(t, 0, \Psi_{03}\right) \Psi_{02}(x)= \\
& =c_{1} \Psi_{1}\left(x, t, \hbar, y_{3}^{(N)}(t, \hbar)\right)+c_{2} \Psi_{2}\left(x, t, \hbar, y_{3}^{(N)}(t, \hbar)\right) .
\end{aligned}
$$

Здесь через $y_{j}^{(N)}(t, \hbar)$ обозначено решение системы Гамильтона-Эренфеста (3.2) порядка $N, N \geqslant 2$, с начальным условием, которое определяется формулами (3.3) по функииям $\Psi_{0 j}(x, \hbar), j=1,2,3$, соответственно.

ПРИЛОЖЕНИЕ

\section{Доказательство свойств 1-4 из раздела 2}

ДокАЗАТЕЛЬСтво СвоЙствА 1. Символ оператора $\{\Delta \hat{z}\}^{\alpha}$ можно записать в виде $(\Delta z)^{\alpha}=(\Delta p)^{\alpha_{p}}(\Delta x)^{\alpha_{x}}, \quad\left(\alpha_{p}, \alpha_{x}\right)=\alpha$, и, следовательно, согласно определению упорядоченных по Вейлю псевдодифференциальных операторов [1] для среднего значения $\sigma_{\alpha}(t, \hbar)$ оператора $\{\Delta \hat{z}\}^{\alpha}$ имеем

$$
\begin{aligned}
\sigma_{\alpha}(t, \hbar)= & \left\langle\Phi\left|\{\Delta \hat{z}\}^{\alpha}\right| \Phi\right\rangle=\frac{1}{(2 \pi \hbar)^{n}} \int_{\mathbb{R}^{3 n}} d x d y d p \Phi^{*}(x, t, \hbar) \times \\
& \times \exp \left(\frac{i}{\hbar}\langle(x-y), p\rangle\right)[\Delta p]^{\alpha_{p}}\left(\frac{\Delta x+\Delta y}{2}\right)^{\alpha_{x}} \Phi(y, t, \hbar),
\end{aligned}
$$

где $\Delta y=y-X(t, \hbar)$. После замены переменных

$$
\Delta x=\sqrt{\hbar} \xi, \quad \Delta y=\sqrt{\hbar} \zeta, \quad \Delta p=\sqrt{\hbar} \omega
$$

с учетом явного вида функций

$$
\Phi(x, t, \hbar)=\exp \left\{\frac{i}{\hbar}(S(t, \hbar)+\langle P(t, \hbar), \Delta x\rangle)\right\} \varphi\left(\frac{\Delta x}{\sqrt{\hbar}}, t, \sqrt{\hbar}\right) \in \mathcal{P}_{\hbar}^{t},
$$

найдем

$$
\begin{aligned}
& \sigma_{\alpha}(t, \hbar)=\frac{1}{(2 \pi \hbar)^{n}} \hbar^{3 n / 2} \hbar^{|\alpha| / 2} 2^{-\left|\alpha_{p}\right|} \int_{\mathbb{R}^{3 n}} d \xi d \zeta d \omega \varphi^{*}(\xi, t, \sqrt{\hbar}) \times \\
& \times \exp \{i\langle\xi-\zeta, \omega\rangle\} \omega^{\alpha_{x}}(\xi+\zeta)^{\alpha_{p}} \varphi(\zeta, t, \hbar)=\hbar^{(n+|\alpha|) / 2} M_{\alpha}(t, \sqrt{\hbar}), \\
& \|\Phi\|^{2}=\hbar^{n / 2} \int_{\mathbb{R}^{n}} d \xi \varphi^{*}(\xi, t, \sqrt{\hbar}) \varphi(\xi, t, \sqrt{\hbar})=\hbar^{n / 2} M_{0}(t, \sqrt{\hbar}) .
\end{aligned}
$$


В силу гладкой зависимости функции $\varphi(\xi, t, \sqrt{\hbar})$ от $\sqrt{\hbar}, \hbar \rightarrow 0$, и того, что $M_{0}(t, \hbar)>0$, отсюда следует

$$
\begin{aligned}
\Delta_{\alpha}(t, \hbar) & =\frac{\sigma_{\alpha}(t, \hbar)}{\|\Phi\|^{2}}=\hbar^{|\alpha| / 2} \frac{M_{\alpha}(t, \sqrt{\hbar})}{M_{0}(t, \sqrt{\hbar})} \leqslant \\
& \leqslant \hbar^{|\alpha| / 2} \max _{t \in[0, T]} \frac{M_{\alpha}(t, \sqrt{\hbar})}{M_{0}(t, \sqrt{\hbar})} \leqslant C(T) \hbar^{|\alpha| / 2}, \quad C(T)>0 .
\end{aligned}
$$

Свойство 2 непосредственно следует из явного вида траекторно-сосредоточенных функций (см. (2.1)) и определения (2.3).

ДокАЗАТЕЛЬСТВо СвоЙСТВА 3. Пусть $\phi(x) \in \mathbb{S}$. Тогда для любой функции $\Phi(x, t, \hbar) \in \mathcal{P}_{\hbar}^{t}$ выражение

$$
\begin{aligned}
\left\langle\frac{|\Phi(t, \hbar)|^{2}}{\|\Phi(t, \hbar)\|^{2}} \mid \phi\right\rangle & =\frac{1}{\|\Phi(t, \hbar)\|^{2}} \int_{\mathbb{R}_{x}^{n}} \phi(x)|\Phi(x, t, \hbar)|^{2} d x= \\
& =\frac{1}{\|\varphi(t, \sqrt{\hbar})\|^{2}} \int_{\mathbb{R}_{x}^{n}} \phi(x)\left|\varphi\left(\frac{\Delta x}{\sqrt{\hbar}}, t, \sqrt{\hbar}\right)\right|^{2} d x
\end{aligned}
$$

после замены переменных $\xi=\Delta x / \sqrt{\hbar}$ преобразуется к виду

$$
\left\langle\frac{|\Phi(t, \hbar)|^{2}}{\|\Phi(t, \hbar)\|^{2}} \mid \phi\right\rangle=\frac{\hbar^{n / 2}}{\|\varphi(t, \sqrt{\hbar})\|^{2}} \int_{\mathbb{R}_{\xi}^{n}} \phi(X(t, \hbar)+\sqrt{\hbar} \xi)|\varphi(\xi, t, \sqrt{\hbar})|^{2} d \xi
$$

Переходя в последнем равенстве к пределу $\hbar \rightarrow 0$, воспользовавшись регулярной зависимостью функции $\varphi(\xi, t, \sqrt{\hbar})$ от $\sqrt{\hbar}$ и тем, что

$$
\|\varphi(t, \sqrt{\hbar})\|^{2}=\hbar^{n / 2} \int_{\mathbb{R}_{\xi}^{n}}|\varphi(\xi, t, \sqrt{\hbar})|^{2} d \xi
$$

получим требуемое утверждение. Доказательство соотношения (2.7) аналогично предыдущему, если заметить, что фурье-образ функции $\Phi(x, t, \hbar) \in \mathcal{P}_{\hbar}^{t}$ имеет вид

$$
\tilde{\Phi}(p, t, \hbar)=\exp \left\{\frac{i}{\hbar}[S(t, \hbar)-\langle p, X(t, \hbar)\rangle]\right\} \tilde{\varphi}\left(\frac{p-P(t, \hbar)}{\sqrt{\hbar}}, t, \sqrt{\hbar}\right),
$$

где

$$
\tilde{\varphi}(\omega, t, \sqrt{\hbar})=\frac{1}{(2 \pi)^{n / 2}} \int_{\mathbb{R}_{\xi}^{n}} e^{-i\langle\omega, \xi\rangle} \varphi(\xi, t, \sqrt{\hbar}) d \xi
$$

ДоКАЗАТЕЛЬСТво СвоЙствА 4 аналогично доказательству соотношений (2.6) и (2.7).

Благодарности. Работа выполнена при частичной финансовой поддержке Российского фонда фундаментальных исследований (грант № 00-01-00087) и Министерства образования РФ (грант № Е00-1.0-126). 


\section{Список литературы}

[1] М. В. Карасев, В. П. Маслов. Нелинейные скобки Пуассона. Геометрия и квантование. М.: Наука, 1991.

[2] С. Р. де Гроот, Л. Г. Сатторп. Электродинамика. М.: Мир, 1982.

[3] С. И. Пекар. Исследования по электронной теории кристаллов. М.: Гостехиздат, 1951.

[4] P. Ring, P. Schuck. The Nuclear Many-Body Problem. New York: Springer, 1980.

[5] J. Kurlandski. Bull. Acad. Pol. Sci. 1982. V. 30. № 3, 4. P. 135.

[6] В. М. Ольхов. ТМФ. 1982. Т. 51. № 1. С. 150.

[7] D. V. Chudnovsky. Infinite component two-dimensional completely integrable systems of KdV type. In: The Riemann Problem, Complete Integrability and Arithmetic Applications. Lect. Notes Math. V. 925. Eds. D. Chudnovsky and G. Chudnovsky. Berlin: Springer, 1982. P. 71.

[8] А. А. Боголюбская, И. Л. Боголюбский. ТМФ. 1983. Т. 54. № 2. С. 258.

[9] D. Gogny, P. L. Lions. J. Math. Phys. 1986. V. 27. № 1. P. 211.

[10] D. R. Hartree. Proc. Cambridge Philos. Soc. 1928. V. 24. P. 89; P. 111; P. 426.

[11] Г. Ефимов. Нелокальное взаимодействие квантованных полей. М.: Наука, 1977.

[12] Ф. А. Березин. Метод вторичного квантования. М.: Наука, 1965.

[13] А. С. Давыцов. Солитоны в молекулярных системах. Киев: Наукова Думка, 1984.

[14] П. Н. Брусов, В. Н. Попов. Сверхтекучесть и коллективные свойства квантовых жидкостей. М.: Наука, 1988.

[15] Y. Lai, H. A. Haus. Phys. Rev. A. 1989. V. 40. P. 844; P. 854.

[16] D. E. Fillips, M. D. Lukin. Phys. Rev. Lett. 2001. V. 86. № 5. P. 783.

[17] A. Zozulya, S. Diddams, T. Clement. Phys. Rev. A. 1988. V. 58. № 4. P. 72.

[18] A. Bove, G. Da Prato, G. Fano. Commun. Math. Phys. 1974. V. 37. P. 183.

[19] J. M. Chadam, R. T. Glassey. J. Math. Phys. 1975. V. 16. P. 1122.

[20] R. T. Glassey. Commun. Math. Phys. 1977. V. 53. № 1. P. 9.

[21] V. Delgado. Proc. Amer. Math. Soc. 1978. V. 69. № 2. P. 289.

[22] I. Fukuda, M. Tsutsumi. J. Math. Anal. Appl. 1978. V. 66. № 2. P. 358.

[23] E. B. Davies. Ann. Inst. H. Poincare. A. 1979. V. 31. № 4. P. 319.

[24] J. Ginibre, G. Velo. Math. Z. 1980. V. 170. № 2. P. 109.

[25] Y. Choquet-Bruhat. C. R. Acad. Sci. Paris. 1981. Ser. 1. V. 292. № 2. P. 153.

[26] W. A. Strauss. J. Funct. Anal. 1981. V. 43. № 3. P. 281.

[27] G. P. Menzala, W. A. Strauss. Diff. Equat. 1982. V. 43. № 1. P. 93.

[28] K. Nakamitsu, M. Tsutsumi. J. Math. Phys. 1986. V. 27. № 1. P. 211.

[29] M. Reeken. J. Math. Phys. 1970. V. 11. P. 2505.

[30] K. Gustafson, D. Sather. Rand. Math. 1971. V. 4. P. 723.

[31] J. Wolkowsky. Indiana Univ. Math. J. 1972. V. 22. P. 551.

[32] C. Stuart. Arch. Rat. Mech. Anal. 1973. V. 51. P. 60.

[33] G. Fonte, R. Mignani, G. Schiffrer. Comm. Math. Phys. 1973. V. 33. P. 293.

[34] E. H. Lieb, B. Simon. Commun. Math. Phys. 1977. V. 53. № 3. P. 185.

[35] E. H. Lieb. Stud. Appl. Math. 1977. V. 57. P. 93.

[36] P. Bader. Proc. Roy. Soc. Edinburgh. A. 1978. V. 82. № 1, 2. P. 27.

[37] G. P. Menzala. On a Hartree type equation: existence of regular solutions. In: Functional Differential Equations and Bifurcations. Lect. Notes Math. V. 799. Ed. A. F. Izé. Berlin: Springer, 1980. P. 277.

[38] G. Rosensteel, E. Ihrig. J. Math. Phys. 1980. V. 21. № 8. P. 2297.

[39] P. L. Lions. Nonlinear Anal.: Theory, Meth., Appl. 1980. V. 4. № 6. P. 1063. 
[40] A. Bongers. Z. Angew. Math. Mech. 1980. V. 60. № 7. P. 240.

[41] J. D. Gegenberg, A. J. Das. J. Math. Phys. 1981. V. 22. № 8. P. 1736.

[42] P. L. Lions. Nonlinear Anal.: Theory, Meth., Appl. 1981. V. 5. № 11. P. 1245.

[43] H. J. Efinger, H. Grosse. Lett. Math. Phys. 1984. V. 8. № 2. P. 91.

[44] P. L. Lions. Comm. Math. Phys. 1987. V. 109. № 1. P. 33.

[45] В. П. Маслов. Комплексные марковские цепи и континуальный интеграл Фейнмана. М.: Наука, 1976.

[46] В. П. Маслов. Уравнения самосогласованного поля. В сб.: Итоги науки и техники. Современные проблемы математики. Т. 11. Ред. Р. В. Гамкрелидзе. М.: ВИНИТИ, 1978. С. 153.

[47] М. В. Карасев, В. П. Маслов. Алгебры с общими перестановочными соотношениями и их приложения. II. Операторные унитарно-нелинейные уравнения. В сб.: Итоги науки и техники. Современные проблемы математики. Т. 13. Ред. Р. В. Гамкрелидзе. М.: ВИНИТИ, 1979. C. 145 .

[48] В. П. Маслов. Комплексный метод ВКБ в нелинейных уравнениях. М.: Наука, 1977.

[49] И. В. Сименог. ТМФ. 1977. Т. 30. № 3. С. 408.

[50] С. А. Вакуленко, В. П. Маслов, И. А. Молотков, А. И. Шафаревич. Докл. РАН. 1995. T. 345 . № 6. C. 743 .

[51] И. А. Молотков, С. А. Вакуленко, М. А. Бисярин. Нелинейные локализованные волновые процессы. М.: Янус-К, 1999.

[52] С. И. Черных. ТМФ. 1982. Т. 52. № 3. С. 491.

[53] М. В. Карасев, А. В. Перескоков. ТМФ. 1989. Т. 79. № 2. С. 198.

[54] М. В. Карасев, А. В. Перескоков. ТМФ. 1993. Т. 97. № 1. С. 78.

[55] М. В. Карасев, А. В. Перескоков. Изв. РАН. Сер. Матем. 2001. Т. 65. № 5. С. 33.

[56] М. В. Карасев, А. В. Перескоков. Изв. РАН. Сер. Матем. 2001. Т. 65. № 6. С. 57.

[57] V. P. Maslov. Russ. J. Math. Phys. 1995. V. 3. № 2. P. 1.

[58] В. П. Маслов, О. Ю. Шведов. Метод комплексного ростка в квантовой теории поля. М.: Изд-во УРСС, 1998.

[59] В. Г. Багров, В. В. Белов, И. М. Тернов. ТМФ. 1982. Т. 50. № 3. С. 390.

[60] V. G. Bagrov, V. V. Belov, I. M. Ternov. J. Math. Phys. 1983. V. 24. № 12. P. 2855.

[61] В. Г. Багров, В. В. Белов, А. Ю. Трифонов. Квазиклассически сосредоточенные состояния уравнения Шредингера. В сб.: Лекционные заметки по теоретической и математической физике. Т. 1. Ч. 1. Ред. А. В. Аминова. Казань: Каз. ГУ, 1996. С. 15.

[62] V. G. Bagrov, V. V. Belov, A. Yu. Trifonov. Ann. Phys. 1996. V. 246. № 2. P. 231.

[63] В. В. Белов, В. П. Маслов. ДАН СССР. 1989. Т. 305. № 3. С. 574.

[64] В. В. Белов, В. П. Маслов. ДАН СССР. 1990. Т. 311. № 4. С. 849.

[65] В. В. Белов, М. Ф. Кондратьева. ТМФ. 1992. Т. 92. № 1. С. 41.

[66] Э. Шредингер. Непрерывный переход от микро- к макромеханике. В сб.: Избр. тр. по квантовой механике. М.: Наука, 1976. С. 51.

[67] R. J. Glauber. Phys. Rev. 1963. V. 130. № 6. P. 2529; V. 131. № 6. P. 2766.

[68] П. К. Рашевский. УМН. 1958. Т. 13. № 3. С. 3.

[69] J. R. Klauder. J. Math. Phys. 1963. V. 4. № 8. P. 1055; P. 1058; 1964. V. 5. № 2. P. 177.

[70] Н. А. Черников. ЖЭТФ. 1967. Т. 53. № 3. С. 1006.

[71] М. А. Малкин, В. И. Манько. Динамические симметрии и когерентные состояния квантовых систем. М.: Наука, 1979.

[72] А. М. Переломов. Обобщенные когерентные состояния и их применение. М.: Наука, 1987.

[73] В. В. Белов. Квазиклассический предел уравнений движения квантовых средних для нерелятивистских систем с калибровочными полями. Препринт № 58. Томск: Томский научный центр СО АН СССР, 1989. 
[74] V. G. Bagrov, V. V. Belov, M. F. Kondratyeva, A. M. Rogova, A. Yu. Trifonov. J. Moscow Phys. Soc. 1993. V. 3. P. 309.

[75] В. Г. Багров, В. В. Белов, М. Ф. Кондратьева. ТМФ. 1994. Т. 98. № 1. С. 48.

[76] В. В. Белов, М. Ф. Кондратьева. Матем. заметки. 1994. Т. 56. № 6. С. 27.

[77] В. В. Белов, М. Ф. Кондратьева. Матем. заметки. 1995. Т. 58. № 6. С. 803.

[78] V. P. Maslov. The Complex WKB Method for Nonlinear Equations. I. Linear Theory. Basel, Boston, Berlin: Birkhauser, 1994.

[79] H. Hayashi, P. I. Naumkin. SUT J. Math. 1998. V. 34. № 1. P. 13.

[80] J. Ginibre, G. Velo. Rev. Math. Phys. 2000. V. 12. P. 361; Ann. Inst. H. Poincare. 2000. V. 1. P. 753 .

[81] В. П. Маслов. Операторные методы. М.: Наука, 1973.

[82] Г. Бейтман, А. Эрдейи. Высшие трансцендентные функции. Функции Бесселя. Функции параболического цилиндра. Ортогональные многочлены. М.: Наука, 1966.

[83] В. И. Арнольд. Математические методы классической механики. М.: Наука, 1983.

[84] В. М. Бабич, В. С. Булдырев. Асимптотические методы в теории дифракции коротких волн. M.: Наука, 1972.

[85] М. Рид, Б. Саймон. Методы современной математической физики. Т. 3. М.: Мир, 1982.

[86] M. М. Попов. Функции Грина уравнения Шредингера с квадратичным потенциалом. В сб.: Пробл. матем. физики. Вып. 6. Ред. В. М. Бабич. Л.: ЛГУ, 1973. С. 119.

[87] V. V. Dodonov, I. A. Malkin, V. I. Man’ko. Int. J. Theor. Phys. 1975. V. 14. № 1. P. 37.

[88] В. В. Белов, Г. Н. Сережников, А. Ю. Трифонов, А. В. Шаповалов. Симметрия и дифференциальные уравнения. В сб.: Тр. междунар. конф. Красноярск, 21-25 августа 2000г. Ред. В. К. Андреев. С. 39.

[89] В. Д. Баранов, В. В. Белов, А. Ю. Трифонов, А. В. Шаповалов. Новейшие проблемы теории поля. В сб.: Тр. Междунар. летней школы-семинара по современным проблемам теор. и матем. физики. Ред. А. В. Аминова. Казань: Каз. ГУ, 2000. С. 22.

[90] Г. Н. Сережников, А. Ю. Трифонов, А. В. Шаповалов. Изв. Томск. гос. ун-та. 2000. T. 1. C. 39. 